\title{
Hierarchical Structure of Azbel-Hofstader Problem: Strings and loose ends of Bethe Ansatz
}

\author{
A.G. Abanov, J.C. Talstra *, P.B. Wiegmann ${ }^{\dagger}$
}

\begin{abstract}
We present numerical evidence that solutions of the Bethe Ansatz equations for a Bloch particle in an incommensurate magnetic field (Azbel-Hofstadter or AH model), consist of complexes - "strings". String solutions are wellknown from integrable field theories. They become asymptotically exact in the thermodynamic limit. The string solutions for the AH model are exact in the incommensurate limit, where the flux through the unit cell is an irrational number in units of the elementary flux quantum.

We introduce the notion of the integral spectral flow and conjecture a hierarchical tree for the problem. The hierarchical tree describes the topology of the singular continuous spectrum of the problem. We show that the string content of a state is determined uniquely by the rate of the spectral flow (Hall conductance) along the tree. We identify the Hall conductances with the set of Takahashi-Suzuki numbers (the set of dimensions of the irreducible representations of $U_{q}\left(s l_{2}\right)$ with definite parity).

In this paper we consider the approximation of noninteracting strings. It provides the gap distribution function, the mean scaling dimension for the bandwidths and gives a very good approximation for some wave functions which even captures their multifractal properties. However, it misses the multifractal character of the spectrum.
\end{abstract}

\section{INTRODUCTION}

The problem of two-dimensional electrons in a periodic potential and a uniform magnetic field (Azbel-Hofstadter problem [1,2]) has a rich history and numerous applications. It is equivalent (in the Landau gauge) to a one-dimensional quasiperiodic difference equation:

$$
\psi_{n+1}+\psi_{n-1}+2 \lambda \cos \left(k_{y}+2 \pi n \eta\right) \psi_{n}=E \psi_{n}
$$

\footnotetext{
*James Franck Institute of the University of Chicago, 5640 S.Ellis Avenue, Chicago, IL 60637, USA

${ }^{\dagger}$ James Franck Institute and Enrico Fermi Institute of the University of Chicago, 5640 S.Ellis Avenue, Chicago, IL 60637, USA and Landau Institute for Theoretical Physics, Moscow, Russia
} 
with two competing incommensurate periods 1 and $1 / \eta$. Here $\eta$ is the flux of the magnetic field through the unit cell in units of the flux quantum $h / e$. This equation is also known as the Harper equation, the almost (or discrete) Mathieu equation, etc. It has been applied to quasicrystals, localization/delocalization transition [3] 5], quantum Hall effect [6] and even DNA chains [7]. For recent review of models of Hofstadter type see [8] and references therein.

The spectrum of this equation is complex. If the flux $\eta$ is a rational number $P / Q$, there is a common period $Q$ in the equation (1i) and the spectrum consists of $Q$ bands. In the incommensurate limit, when $\eta$ is an irrational number $(P \rightarrow \infty, Q \rightarrow \infty)$ the spectrum becomes an infinite Cantor set [1,9] with total bandwidth (Lebesgue measure of the spectrum) $4|\lambda-1|[3,[10,[1]$. At $|\lambda|=1$ the spectrum becomes a purely singular continuous - uncountable but measure zero set of points [12 (for review see [13,5]). There is numerical evidence that in this case the spectrum and wave functions are multifractal [14.

The scaling description of multifractal sets, generated by a quasiperiodic equation, an important and challenging problem, is far from being understood. The reasons are twofold: a lack of language to describe scaling properties of singular continuous sets and a lack of methods to attack incommensurate systems.

Recently, it has been shown that the Harper equation, belongs to the class of integrable models of quantum field theory. Despite being just a one particle problem, it has been "solved" by the Bethe Ansatz [15]. This creates a perspective for an analytical solution of the problem and eventually for the employment of methods of conformal field theory for finding scaling properties of multifractal sets.

Below we make a first step towards solving the Bethe Ansatz equations for the most interesting critical case $|\lambda|=1$. We show that in the incommensurate limit roots of the Bethe Ansatz equations are grouped in complexes called strings, so that each state is a composition of strings. Strings are well known for standard integrable models of quantum field theory, for instance XXZ Heisenberg chain [17. They become exact only for a macroscopic system, where the number of particles and the size of the system are sent to infinity. The role of thermodynamic limit for the AH problem is obviously the incommensurability $-P, Q \rightarrow \infty$, $P / Q \rightarrow \eta=$ irrational. The common period $Q$ plays the role of the size of the system. Indeed, we present numerical evidence that the "string hypothesis" remains valid - strings become exact in the incommensurate limit $Q \rightarrow \infty$.

A first important application of the string structure of solutions is a detailed hierarchical tree of the spectrum, i.e. an algorithm for generating this Cantor set spectrum [18. The

\footnotetext{
${ }^{1}$ Although some properties of the spectrum depend on the type of irrational number $\eta$, in this paper we consider the so called typically Diophantine numbers. These numbers have a full Lebesgue measure and thus suffice for almost all physical applications.

${ }^{2}$ closed, nowhere dense set which has no isolated points

${ }^{3}$ The initial progress toward solving Bethe equations of [15 has been made in 16] where the explicit analytical solution for zero energy level as well as some numerical results for midband levels are reported.
} 
hierarchical tree gives the topology of the set (Sec. [V]). We show that the string decomposition of a state is tied to the holonomy of the wave function, i.e. the Hall conductance of the state, and therefore must be of an algebraic geometrical nature. The set of Hall conductances generates the spectral flow, which in its turn describes the hierarchical tree (Sec. [I]).

Since strings become exact in the incommensurate limit with an accuracy $\mathcal{O}\left(Q^{-2}\right)$, at large but finite $\mathrm{Q}$, the "bare" noninteracting strings give a very good numerical approximation for some wave functions. This approximation even captures the multifractal properties of wave functions (Sec. $\nabla \mathrm{I})$ ). The noninteracting strings give rise to a distribution function of gaps of the spectrum (Sec. $\nabla \mathrm{A}$ ). The string hypothesis also gives a mean field scaling exponent for the spectrum (bandwidth distribution), and provides a framework for calculating anomalous dimensions as "finite size" corrections to the bare values obtained at $Q \rightarrow \infty$ from string solutions. We will not compute them in this paper.

We start by the construction of the hierarchical tree for the spectral flow of a general quasiperiodic system (Sec. [II). Then in Sec. III we review the Bethe Ansatz for the AH problem and formulate the string solution. In Sec. $\mathbb{\square}$ we argue that string solutions are tied to the Hall conductances and generate the hierarchical tree described in Sec. II. The string hypothesis is used to compute the gap and bandwidth distributions in (Sec. $\nabla \mathrm{A}$ and Sec. VB). We conclude with a list of results and "loose ends". In the Appendix (Sec. [X] we present a technically involved derivation of Bethe Ansatz equations.

\section{HIERARCHICAL-TREE: SPECTRAL FLOW, HALL CONDUCTANCE AND INTERMEDIATE FRACTIONS.}

The hierarchical tree is an adequate language to describe properties of quasiperiodic systems and strange sets these systems generate. In this section we describe the hierarchical tree for the spectrum of the AH problem, although a major part of the construction seems to be valid for more general quasiperiodic linear systems. Later in Sec. IV we show that this tree determines the structure of the Bethe roots as well.

The hierarchical tree may be defined as follows. Let us consider a sequence of rational approximants $\eta^{(j)}=P^{(j)} / Q^{(j)}$ with increasing $Q^{(j)}$ to an irrational flux $\eta$ so that: $\left|\eta^{(j)}-\eta\right|<$ $c\left(Q^{(j)}\right)^{-2}$, where $c$ is $j$-independent constant . We refer to the spectrum of the Harper equation characterized by the flux $\eta^{(j)}=P^{(j)} / Q^{(j)}$ as the generation of the hierarchy. Let us connect the $k$-th band of the generation $\eta^{(r)}$ (the daughter generation) to a certain band $k^{\prime}$ of some previous (parent) generation $\eta^{\left(r^{\prime}\right)}$, thus creating an infinite graph with no loops. We call this graph a hierarchical tree if energies $E_{j}(\mathcal{J})$ belonging to any branch $\mathcal{J}$ of the tree form a sequence converging to the point $E(\mathcal{J})$ of the spectrum in such a way that the sequence $\left(Q^{(j)}\right)^{2-\epsilon_{\mathcal{J}}}\left|E_{j}(\mathcal{J})-E(\mathcal{J})\right|$ is bounded but does not converge to zero. The numbers $\epsilon_{\mathcal{J}}$ are anomalous exponents

\footnotetext{
${ }^{4}$ This sequence always exists and can be constructed e.g. from the so called Farey Series [21]

${ }^{5}$ To avoid confusion let us stress that the very existence of the hierarchical tree is a (scaling)
} 
Fig. 1. To construct the hierarchical tree it is necessary to find the sequence of generations and a rule to determine the parent band out of a given band of a given generation.

Below we conjecture that the hierarchical tree is the spectral hierarchy - an integral version of the spectral flow. Let us recall the notion of spectral flow and its rate - the Hall conductance. Consider the spectrum of the problem (1) for a given flux $\eta^{(r)}=P / Q$. It consists of $\mathrm{Q}$ bands separated by gaps. Let us choose some gap $k$ and change the flux by an infinitesimal $\delta \eta$. The spectrum remains unchanged except for some new levels that appear within a gap. The number $\delta N_{k}$ of levels crossing the energy $E$, lying inside the gap, is a spectral flow. Its rate

$$
\sigma_{k}=\frac{\delta N_{k}}{\delta \eta}
$$

is known to be the Hall conductance of the gap [22]. This number is an integer and depends on the gap. The difference between the Hall conductances of neighboring gaps, i.e. the spectral flow into the $k$-th band $\sigma(k)=\sigma_{k}-\sigma_{k-1}$ is the Hall conductance of the band.

Let us now consider two close rational numbers $\eta^{(j)}=P / Q$ and $\eta^{(j-1)}=P^{\prime} / Q^{\prime}$ with $Q^{\prime}<Q$. The number of states in a band is $1 / Q$ and $1 / Q^{\prime}$ respectively in units of $N$, where $N$ is the total number of lattice sites. If there is a band $k$ of the problem with the flux $P / Q$, and we can find a flux $P^{\prime} / Q^{\prime}$ with $Q>Q^{\prime}, P \geq P^{\prime}$ such that its Hall conductance is a ratio between the difference of the number of states and the fluxes

$$
\frac{1}{Q}-\frac{1}{Q^{\prime}}=\sigma(k)\left(\frac{P}{Q}-\frac{P^{\prime}}{Q^{\prime}}\right)
$$

then we say that the band $k$ of the generation $P / Q$ has a "parent" band in the generation $P^{\prime} / Q^{\prime}$. This formula may be viewed as an integrated Streda formula (2). It determines the flux $P^{\prime} / Q^{\prime}$ and by virtue of an iterative procedure, generates a sequence of rational approximants (generations) $\eta^{(j)}$ to the flux $\eta$. The sequence $\eta^{(j)}$ differs from the approximants obtained by truncating the continued fraction expansion of

$$
\eta=\frac{1}{n_{1}+\frac{1}{n_{2}+\frac{1}{n_{3}+\ldots}}} \equiv\left[n_{1}, n_{2}, n_{3}, \ldots\right] .
$$

Instead it consists of so called intermediate fractions approximants (see below) 223.

We complete the integrated Streda formula by the adiabatic principle: let us enumerate all states from the bottom of the spectrum and characterize them by an integrated density of states (IDS) $\nu=N_{\text {states }} / Q$. Here $N_{\text {states }}$ is a total number of states with energy less than the energy of the given state. All states in the $k$-th band have the IDS $(k-1) / Q<\nu<k / Q$.

hypothesis. Moreover, the tree constructed in this paper is a conjecture. There is no deeper reason for the hypothesis and the conjecture other than physically plausible arguments and numerical evidence. 
The adiabatic principle assumes that a middle state 9 in the band $k$ with IDS $(k-1 / 2) / Q$ has a parent in the parent band $k^{\prime}$ such that $\left(k^{\prime}-1\right) / Q^{\prime}<(k-1 / 2) / Q<k^{\prime} / Q^{\prime}$. This gives

$$
k^{\prime}=\left[\frac{Q^{\prime}}{Q}\left(k-\frac{1}{2}\right)\right]+1
$$

and together with integrated Streda formula determines the hierarchical tree. $[x]$ denotes the integer part (floor function) of $x$.

To construct the tree, we need to know the Hall conductance of a band $\sigma(k)$ and the Hall conductance of a gap $\sigma_{k}$. The Hall conductivity of the $k$-th gap at generation $P / Q$ is known to be a solution of the Diophantine equation [24,25]

$$
P \sigma_{k}=k(\bmod Q)
$$

restricted to the range $-Q / 2<\sigma_{k} \leq Q / 2$.

To write the solution of this equation, let us represent the flux $\eta^{(j)}=P / Q$ of the given generation as a continued fraction (14) and denote the convergents $P_{i} / Q_{i}=$ $\left[n_{1}, n_{2}, \ldots n_{i}\right], \quad i=1, \ldots r ; P_{r} / Q_{r} \equiv P / Q=\eta^{(j)}$. Then the Hall conductance of a gap is

$$
\sigma_{k}=\frac{Q_{r}}{2}-Q_{r}\left\{(-1)^{r} \frac{Q_{r-1}}{Q_{r}} k+\frac{1}{2}\right\}
$$

where $\{x\}$ is the fractional part of $x$.

In its turn the Hall conductivity carried by the $k$-th band $\sigma(k)=\sigma_{k}-\sigma_{k-1}$. It is easy to see that the Hall conductivity of the band takes only two values $(-1)^{r-1} Q_{r-1}$ and $(-1)^{r}\left(Q-Q_{r-1}\right)$. It determines the parent generation by virtue of (3) $Q^{\prime}=Q-|\sigma(k)|$ :

$$
\eta^{(j-1)}=\frac{P^{\prime}}{Q^{\prime}}=\left\{\begin{array}{lll}
\frac{P_{r}-P_{r-1}}{Q_{r}-Q_{r-1},}, & \text { if } \quad \sigma(k)=(-1)^{r-1} Q_{r-1} \\
\frac{P_{r-1}}{Q_{r-1}}, & \text { if } \quad \sigma(k)=(-1)^{r}\left(Q_{r}-Q_{r-1}\right)
\end{array}\right.
$$

This formula looks better in terms of the continued fraction

$$
\eta^{(j-1)}= \begin{cases}{\left[n_{1}, n_{2}, \ldots, n_{r}-1\right],} & \text { if } \sigma(k)=(-1)^{r-1} Q_{r-1} \\ {\left[n_{1}, n_{2}, \ldots, n_{r-1}\right],} & \text { if } \sigma(k)=(-1)^{r}\left(Q_{r}-Q_{r-1}\right)\end{cases}
$$

Thus the parent generation is obtained by either subtracting 1 from the last quotient $n_{r}$ of the continued fraction or by truncating the fraction. The sequence of generations produced by these iterations is known as the intermediate fractions. These numbers are defined as numbers of the type $\frac{P_{i-2}+\mu P_{i-1}}{Q_{i-2}+\mu Q_{i-1}}$ with $\mu=0,1,2, \ldots, n_{i}$. The intermediate fractions list all the best rational approximants of the number $\eta$ [26]. This is sufficient to construct the hierarchical tree.

\footnotetext{
${ }^{6}$ Middle state is the state for which the number of states in the given band with lower energy is the same as number of ones with higher energy.
} 
Applying the same procedure to the parent generation we obtain another set of values of Hall conductances of bands. The entire set of Hall conductances generated by the flux $P / Q$ are numbers less than $Q$ of the form

$$
\left\{Q_{i-2}+m Q_{i-1} \mid m=1, \ldots, n_{i}, \quad i=1,2, \ldots, r\right\} .
$$

This set consists of numbers (denominators of intermediate fractions) known in integrable models related to $U_{q}\left(S L_{2}\right)$ as Takahashi-Suzuki numbers [17]. They are allowed lengths of strings in solutions, say for the XXZ model. These numbers are also the set of possible dimensions of irreducible highest weight representations of $U_{q}\left(S L_{2}\right)$ with definite parity [27]. We might have expected these numbers to play a role, considering that the intimate connection between $U_{q}\left(S_{2}\right)$ and the AH-problem has already been outlined in previous publications [15].

Each path of the tree may also be characterized by a sequence of fractional numbers of bands: $\nu_{k}^{(j)}=k / Q^{(j)}$, lying on the path and converging to a given irrational fraction $\nu$. According to eq.(5) the parent fraction is determined by the daughter one as $\nu^{(j-1)}=$ $\frac{1}{Q^{(j-1)}}\left(\left[Q^{(j-1)}\left(\nu^{(j)}-\frac{1}{2 Q^{(j)}}\right)\right]+1\right)$ with $Q^{(j-1)}=Q^{(j)}-|\sigma(k)|$. The sequence $\nu^{(j)}$ converges to the irrational $\nu$ faster than $\left(Q^{(j)}\right)^{-1},\left|\nu^{(j)}-\nu\right|<c\left(Q^{(j)}\right)^{-1}$. In terms of the fractions one may reformulate the scaling hypothesis as $\left|E_{j}-E\right|<c\left|\nu^{(j)}-\nu\right|^{\alpha(\mathcal{J})}$, defining the scaling exponent $\alpha(\mathcal{J})$.

To illustrate the construction of the hierarchical tree described above, let us consider some particular flux, say, $\eta=P / Q=4 / 15=[3,1,3]$. The spectrum of AH problem for this flux consists of 15 bands and 14 gaps. The ancestral generations of the tree are given by the sequence is $\frac{0}{1}, \frac{1}{2}, \frac{1}{3}, \frac{1}{4}, \frac{2}{7}, \frac{3}{11}, \frac{4}{15}$. Notice, that the truncated continuum fractions are different. They are $\frac{0}{1}, \frac{1}{3}, \frac{1}{4}, \frac{4}{15}$ just a subset of generations. The Hall conductances of bands and gaps (ordered according to their energy) for each generation are:

$$
\begin{aligned}
\eta & =\frac{4}{15}: \begin{cases}\sigma(k) & =4,-11,4,4,4,-11,4,4,4,-11,4,4,4,-11,4 \\
\sigma_{k} & =4,-7,-3,1,5,-6,-2,2,6,-5,-1,3,7,4\end{cases} \\
\eta_{6} & =\frac{3}{11}: \begin{cases}\sigma(k) & =4,-7,4,4,-7,4,-7,4,4,-7,4 \\
\sigma_{k} & =4,-3,1,5,-2,2,-5,-1,3,-4\end{cases} \\
\eta_{5} & =\frac{2}{7}:\left\{\begin{array}{l}
\sigma(k)=-3,4,-3,4,-3,4,-3 \\
\sigma_{k}=-3,1,-2,2,-1,3
\end{array}\right. \\
\eta_{4} & =\frac{1}{4}: \begin{cases}\sigma(k) & =1,1,-3,1 \\
\sigma_{k} & =1,2,-1\end{cases} \\
\eta_{3} & =\frac{1}{3}: \begin{cases}\sigma(k) & =1,-2,1 \\
\sigma_{k} & =1,-1\end{cases} \\
\eta_{2} & =\frac{1}{2}: \begin{cases}\sigma(k) & =1,-1 \\
\sigma_{k} & =1\end{cases}
\end{aligned}
$$

One should notice the asymmetry of $\sigma(k)$ for $\eta_{4}$. This asymmetry is related to the degeneracy present for the fluxes with even denominators (two bands touch each other at energy $E=0$ ). The two possible choices $\sigma_{2}= \pm 2$ for the Hall conductance in the "gap" at $E=0$ (actually there is no gap) restore the symmetry of the tree with respect to $E \rightarrow-E$. This tree, 
depicted in Fig.1 (half of the tree is shown) consists of 15 non-crossing branches $J_{i}, i=$ $1,2, \ldots 15$. They connect bands, characterized by the flux and number of the band, with Hall conductances $\sigma(k)$ as listed below. The branches are listed in order of increasing energy. Only the lower half of the spectrum is presented

$$
\begin{aligned}
& J_{1}=\left(\frac{1}{2}, 1,1 ; \frac{1}{3}, 1,1 ; \frac{1}{4}, 1,1 ; \frac{2}{7}, 1,-3 ; \frac{3}{11}, 1,4 ; \frac{4}{15}, 1,4\right), \\
& J_{2}=\left(\frac{1}{2}, 1,1 ; \frac{1}{3}, 1,1 ; \frac{1}{4}, 1,1 ; \frac{4}{15}, 2,11\right), \\
& J_{3}=\left(\frac{1}{2}, 1,1 ; \frac{1}{3}, 1,1 ; \frac{1}{4}, 1,1 ; \frac{3}{11}, 2,-7 ; \frac{4}{15}, 3,4\right), \\
& J_{4}=\left(\frac{1}{2}, 1,1 ; \frac{1}{3}, 1,1 ; \frac{2}{7}, 2,4 ; \frac{3}{11}, 3,4 ; \frac{4}{15}, 4,4\right), \\
& J_{5}=\left(\frac{1}{3}, 2,-2 ; \frac{1}{4}, 2,1 ; \frac{2}{7}, 3,-3 ; \frac{3}{11}, 4,4 ; \frac{4}{15}, 5,4\right), \\
& J_{6}=\left(\frac{1}{3}, 2,-2 ; \frac{1}{4}, 2,1 ; \frac{4}{15}, 6,-11\right), \\
& J_{7}=\left(\frac{1}{3}, 2,-2 ; \frac{1}{4}, 2,1 ; \frac{3}{11}, 5,-7 ; \frac{4}{15}, 7,4\right), \\
& J_{8}=\left(\frac{1}{3}, 2,-2 ; \frac{2}{7}, 4,4 ; \frac{3}{11}, 6,4 ; \frac{4}{15}, 8,4\right) .
\end{aligned}
$$

Here in brackets we showed the flux, the number of the band and the Hall conductance of this band.

\section{BETHE ANSATZ EQUATIONS AND THE STRING HYPOTHESIS}

The Bethe Ansatz solution is available for any point of the Brillouin zône $0 \leq k_{x}<$ $2 \pi / Q, 0 \leq k_{y}<2 \pi(\eta=P / Q)$ [28], but it looks especially simple in the rational points of the Brillouin zône (the definition of rational points and corresponding values of $k_{x}$ and $k_{y}$ are given in Sec. [X]). They correspond to the centers and edges of bands. The study of these points is sufficient for our purposes?. Below we sketch the results of the Bethe-Ansatz solution. The details can be found in the Appendix and also in Refs. 115,28,29. The Harper eq. (1) at rational points can be mapped onto a functional equation

$$
\begin{aligned}
\mu \kappa z E \Psi(z)= & i q\left(z+i \tau \kappa q^{-\frac{1}{2}}\right)\left(z-i \kappa q^{-\frac{1}{2}}\right) \Psi(q z) \\
& -i q^{-1}\left(z-i \tau \kappa q^{\frac{1}{2}}\right)\left(z+i \kappa q^{\frac{1}{2}}\right) \Psi\left(q^{-1} z\right)
\end{aligned}
$$

where $q=e^{i \pi \eta}$ and $\tau, \kappa, \mu= \pm$ are labels of the rational points (see Sec. IX).

The wave function of the original problem (1) can be obtained as a linear combination of $\psi_{n}=\Psi\left(-e^{-i p_{-}} q^{n}\right)$ where the momentum $p_{-}$belongs to a set of rational points of the Brillouin zône. For $P=$ odd the rational points correspond to the center of a band. There are two distinct sets - one for $\tau=+1$, another for $\tau=-1$. For $P=$ even and $\tau=1$ a rational point also corresponds to a center of the band. The rational points at $\tau=-1$ and $P=$ even, give bottom edges of odd bands and top edges of even bands for $\mu(-1)^{P / 2}=-1$, counted from the bottom of the spectrum and top edges of odd bands and bottom edges of even bands for $\mu(-1)^{P / 2}=+1$. This allows one to find bandwidths and gaps. Let us take $P=$ even and numerate the energy levels corresponding to rational points with $\tau=-1$ according to their order starting from the bottom of the spectrum $E_{n}, n=1, \ldots, Q$. Then the width of the $n$-th band $\left(W_{n}\right)$ and the $n$-th gap $\left(D_{n}\right)$ are:

\footnotetext{
${ }^{7}$ We also restrict ourselves to the isotropic (critical) case $\lambda=1$.
} 


$$
D_{n}=\mu\left\{\begin{array}{ll}
W_{n}=\mu(-1)^{\frac{P}{2}+n-1}\left(E_{n}+E_{Q+1-n}\right), \\
E_{n+1}-E_{n} & \text { if } P / 2+n \text { - odd } \\
E_{Q-n+1}-E_{Q-n} & \text { if } P / 2+n \text { - even }
\end{array} .\right.
$$

For $P$-odd and $Q$-odd edges of bands and their widths may be obtained by the flux reflection $q \rightarrow-q^{-1}$.

The integrability manifests itself in the fact that the solutions to the difference equation (12) are polynomials

$$
\Psi(z)=\prod_{i=0}^{Q-1}\left(z-z_{i}\right)
$$

with roots obeying Bethe equations:

$$
q^{Q} \prod_{j=1}^{Q-1} \frac{q z_{i}-z_{j}}{z_{i}-q z_{j}}=\frac{\left(z_{i}-i \tau \kappa q^{\frac{1}{2}}\right)\left(z_{i}+i \kappa q^{\frac{1}{2}}\right)}{\left(q^{\frac{1}{2}} z_{i}+i \tau \kappa\right)\left(q^{\frac{1}{2}} z_{i}-i \kappa\right)} .
$$

These equations have $Q$ independent solutions. Each of them consists of $Q-1$ complex numbers $\left\{z_{1}, \ldots, z_{Q-1}\right\}$ and corresponds to an eigenstate of the Harper equation at a rational point of the Brillouin zône with the energy:

$$
E=i \mu q^{Q}\left(q-q^{-1}\right) \frac{1}{2}\left[\kappa \sum_{i=1}^{Q-1}\left(z_{i}-q^{-Q} \tau z_{i}^{-1}\right)-i \frac{\left(1-q^{-Q}\right)(1-\tau)}{q^{1 / 2}-q^{-1 / 2}}\right]
$$

The Bethe equations (15) are similar to the equations for the XXZ chain with maximal allowed spin $S=\frac{Q-1}{2}$ and commensurate anisotropy $\cos \left(\frac{\pi}{2} \eta\right)$. The major difference is that it corresponds to a spin-problem on one or two sites [15, 30].

Below we give evidence that in an incommensurate limit all Bethe roots are grouped to strings. Each string of the "spin" $l$ is a complex of $2 l+1$ roots

$$
\left\{z_{m}^{(l)}=v_{l} x_{l} q_{l}^{m}, \quad m=-l,-l+1 \ldots, l\right\}
$$

which have a common modulus $x_{l}>0$ (the center of the string), a parity $v_{l}= \pm 1$ and differ by multiples of $q_{l}$. We show that $q_{l}$ is the $2 l+1$-th root of unity, "closest" to $q$ (Sec. [V] and $x_{l}=1+\mathcal{O}\left(Q^{-1}\right)$.

Each state is characterized by a set of spins $\left\{l_{k}, l_{k-1}, \ldots\right\}$ and parities $\left\{v_{k}, v_{k-1}, \ldots\right\}$ of the string content, such that the total number of roots $\sum_{i=1}^{k}\left(2 l_{i}+1\right)=Q-1$

$$
\Psi^{\left\{l_{k}, v_{k}, \ldots\right\}}(z) \approx \prod_{m=-l_{k}}^{l_{k}}\left(z-x_{l} v_{l} q_{l_{k}}^{m}\right) \Psi^{\left\{l_{k-1}, v_{k-1}, \ldots\right\}}(z) .
$$

Moreover, in the next section we argue that the wave function with a "subtracted string" $\Psi^{\left\{l_{k-1}, v_{k-1}, \ldots\right\}}(z)$ is approximately the wave function of the $\mathrm{AH}$ problem of the parent generation, i.e. the problem with flux $\eta^{\prime}=\eta^{(j-1)}(9)$ and the length of the "subtracted" string is the Hall conductance $l_{k}=|\sigma(k)|$. The formula (17) is asymptotically exact with accuracy increasing with the length of the string as $1 /\left(2 l_{k}+1\right)^{2}$. 
Comparison with the XXZ chain may be useful. In that case strings become exact in the thermodynamical limit, i.e. in the limit where the size of the chain and number of spins up go to infinity faster than anisotropy angle $P / Q$ approaches an irrational number. In that case spins (i.e. lengths) of the strings and their parities are drawn from a subset of integers, called Takahashi-Suzuki numbers, while centers are continuously distributed on the real axis. Our case is different - the number of sites on the equivalent chain is 2. Also one does not expect to describe strange sets by continuous distributions. Nevertheless, the string hypothesis remains robust. Naturally the incommensurability plays the role of "thermodynamical limit". In the Sec. $\mathbb{D}$ we show that allowed spins of strings remain to be the Takahashi-Suzuki numbers, however the number of strings with a given spin is finite and their centers approach 1 with increasing length of the string.

Strings play a bigger role than just being solutions of the Bethe equations. The spins of strings of which the state is composed give the topological characteristics of the state, related to the holonomy of the wave function in the Brillouin zône (the Hall conductance). They eventually describe the topology of the spectrum.

\section{STRINGS AND HIERARCHY TREE}

In this section we present heuristic arguments for the string hypothesis and support them with numerics in Sec. VI. The arguments are based on the analysis of singularities of the Bethe equations (15). It turns out that the string solutions follow the hierarchical tree of Sec. III.

Let us assume that a solution of the Bethe equations (15) contains a long string of spin $l \gg 1$ (17). Let us substitute this string into (15) and drop all terms, which are not singular as $q_{l} \rightarrow q$. Equations for the first and the last root of the string $v_{l} x_{l} q_{l}^{-l}$ and $v_{l} x_{l} q_{l}^{l}$ give

$$
\begin{aligned}
& \kappa x_{l} v_{l} q_{l}^{-l}\left(q-q_{l}\right)(\ldots) \sim\left(x_{l} v_{l} q_{l}^{-l}-i \kappa q^{\frac{1}{2}} \tau\right)\left(x_{l} v_{l} q_{l}^{-l}+i \kappa q^{\frac{1}{2}}\right) \\
& \kappa x_{l} v_{l} q q_{l}^{l-1}\left(q_{l}-q\right)(\ldots) \sim\left(q^{\frac{1}{2}} x_{l} v_{l} q_{l}^{l}+i \kappa \tau\right)\left(q^{\frac{1}{2}} x_{l} v_{l} q_{l}^{l}-i \kappa\right) .
\end{aligned}
$$

where by $(\ldots)$ we denote the terms of the order one at $q_{l} \rightarrow q$. Compatibility of these equations requires

$$
\left|q_{l}^{2 l} q \pm 1\right|=\mathcal{O}\left(\left|q-q_{l}\right|\right)
$$

If $q_{l}=e^{i \pi P^{\prime \prime} / Q^{\prime \prime}}$ is sufficiently close to $q=e^{i \pi \eta}$ i.e. $\left|\eta-P^{\prime \prime} / Q^{\prime \prime}\right| \sim 1 / Q^{\prime \prime 2}$, Eq. (21) may be satisfied only if

$$
q_{l}^{2 l+1}=\mp 1
$$

where $\mp$ corresponds to the sign in (21). This gives $Q^{\prime \prime}=2 l+1$. We will refer to the strings with $q_{l}^{2 l+1}=+1$ as to "closed" strings as opposed to "open" ones with $q_{l}^{2 l+1}=-1$.

For open strings the equations $(19,20)$ give an approximate value of the center $x_{l} \approx 1$ and two possible values of parity:

$$
\begin{aligned}
& v_{l}=-i q_{l}^{l+1 / 2} \kappa=(-1)^{[\eta l]} \kappa, \\
& v_{l}=+i q_{l}^{l+1 / 2} \tau \kappa=-(-1)^{[\eta l]} \tau \kappa .
\end{aligned}
$$


The symmetries of the Bethe equations require a center to be a real positive number. In case $x_{l} \neq 1$, there is always another string with an inverted center $x_{l}^{-1}$.

If $q_{l}^{2 l+1}=1$ the leading singular terms (19,20) of the Bethe equations vanish. The center and the parity of the closed string are determined by the next leading singularities. We do not study these here. Empirically we still find that centers of closed strings are close to 1 as for open strings.

Now consider the Bethe equation for a root which does not belong to the string. Then, assuming $q_{l}$ to be very close to $q$ after a set of cancelations, we obtain again the Bethe equations for $Q-2 l-2$ remaining roots. If $q_{l}^{2 l+1}=1$ the equations retain their form. In case $q_{l}^{2 l+1}=-1$ the Bethe equations for the point $(\tau, \kappa, \mu)$ are transformed into ones for the point $(-\tau,-\kappa, \mu)$ for a string with the parity (23) and to the point $(-\tau, \kappa,-\mu)$ for the parity (24).

In other words, while subtracting the string from a wave function of a generation where rational points correspond to edges of bands ( $P$ is even) we obtain the Bethe equation for the generation where rational points are the centers of bands $\left(P^{\prime}\right.$ is odd). However, subtracting a string from the generation where rational points are centers ( $P$ is odd) one may get $P^{\prime}$ both even and odd, depending on the sign in eq.(22).

Now comes the crucial step: we have to determine what values $2 l+1$, the length of the string and its parity can take. The new approximate Bethe equations on the remaining $Q-2 l-2$ roots would be consistent if one replaces $q$ by the closest $q^{\prime}$ such that $\left(q^{\prime}\right)^{Q-2 l-1}=$ \pm 1 . This requirement and also the requirement that $q_{l}$ is also the closest to $q$, leaves us with two possibilities

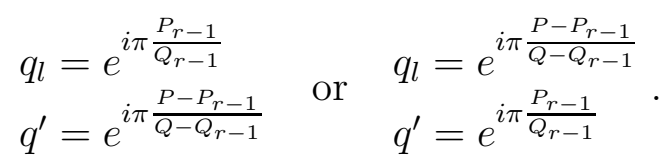

This means that the states of generation $P / Q$ have the largest string of the length

$$
2 l+1=\left\{Q-Q_{r-1} \quad \text { or } \quad Q_{r-1}\right\}
$$

The parent generation of a state may have a flux:

$$
\eta^{(r-1)}=\frac{P_{r-1}}{Q_{r-1}} \quad \text { or } \quad \frac{P-P_{r-1}}{Q-Q_{r-1}}
$$

respectively. Thus we see that the lengths of two possible highest strings are the same as two possible values of Hall conductance of bands (8). We conjecture (and checked this numerically) that the length of the highest string is equal to the Hall conductance of the band.

Now let us discuss the parities of open strings. If $\tau=-1$ the both formulae (23,24) give the same result $v=(-1)^{[\eta l]} \kappa$. However for $\tau=+1$ (center of bands) there is a choice between two possible values of parity. To make this choice we must further specify the hierarchical tree and the spectral flow. Let us consider for example the band $k$ which belongs to the generation $\eta=P / Q$ with $P$-odd and $\tau=+1$. Then the corresponding parent band $k^{\prime}$ belongs to the generation $\eta^{\prime}=P^{\prime} / Q^{\prime}$ with $P^{\prime}$-even (we consider the case when the largest string is open) and $\tau^{\prime}=-\tau=-1$. There are two different energy levels corresponding to 
the rational points in Brillouin zône which belong to the parent band $k^{\prime}$. These are top and bottom of this band. We will call the state corresponding to the top (or bottom) of the band $k^{\prime}$ as parent state of the state in the middle of the $k$-th band if the Hall conductance in the gap adjacent to the top (bottom) of the band $k^{\prime}$ is the same as the one in the gap adjacent to the top (bottom) of the band $k$ of the generation $\eta$. If one goes from the generation $\eta^{\prime}$ to generation $\eta$ in $\mathrm{AH}$ problem only new gaps open in the spectrum while the old ones present in parent generation stay and have the same Hall conductance in the daughter generation. Therefore among two states at the top and the bottom of the parent band we call the parent state the one which is not splitted of from the band by new gap while evolving from the generation $\eta^{\prime}$ to the generation $\eta$. The both states can be parent states if the band $k^{\prime}$ is not split by a new gap.

The edge of the band $k^{\prime}$ corresponding to the rational point characterized by $\kappa^{\prime}$ and $\mu^{\prime}$ is a top of the band if $(-1)^{P^{\prime} / 2+k^{\prime}} \mu^{\prime}=-1$ and it the bottom if $(-1)^{P^{\prime} / 2+k^{\prime}} \mu^{\prime}=+1$. Using the rules of transformation of $\kappa$ and $\mu$ while subtracting open string from the state in the middle of the band $k$ one can easily obtain that the right choice of parity is $v_{l}=\mu \kappa(-1)^{P^{\prime} / 2+k^{\prime}+[\eta l]}$ if the parent state is the bottom of the band $k^{\prime}$ and $v_{l}=-\mu \kappa(-1)^{P^{\prime} / 2+k^{\prime}+[\eta l]}$ if it is the top. Finally, the $k$-th gap at the generation $\eta$ is an old one (present at the generation $\eta^{\prime}$ ) if corresponding Hall conductance $\left|\sigma_{k}\right| \leq Q^{\prime} / 2$. and we obtain:

$$
v_{l}=\mu \kappa(-1)^{P^{\prime} / 2+k^{\prime}+[\eta l]}\left\{\begin{array}{lll}
+1 & \text { if } & \left|\sigma_{k-1}\right| \leq Q^{\prime} / 2 \\
-1 & \text { if } & \left|\sigma_{k}\right| \leq Q^{\prime} / 2
\end{array}\right.
$$

If both of formulae in (28) work one can choose parity to be $v= \pm 1$. We notice than in this case the string content of the state in the middle of the band $k$ must include the string of the largest length at least twice with both possible parities.

Summing up we obtain the iterative procedure of constructing the string decomposition:

- Starting from a band of the generation $\eta^{(r)}=P_{r} / Q_{r}$ and a point of the Brillouin zône $\tau, \kappa, \mu$, we determine the string with highest spin (26). Its parity is given by (23). Subtracting this string we obtain the Bethe equation for a generation $\eta^{(r-1)}$ (27) for a point of the Brillouin zône $\tau^{\prime}=q_{l}^{2 l+1} \tau, \kappa^{\prime}= \pm \kappa$ and so on. Continuing this procedure to the end of the tree we obtain an unambiguous string decomposition of each level.

To illustrate the iterative hierarchical procedure, let us denote by $\left[\frac{P}{Q}, \tau, \kappa, \mu\right]$ the set of roots $(Q-1$ complex numbers) for flux $P / Q$ and rational points of a particular band labeled by $\tau, \kappa$ and $\mu$. Then the iterative procedure can be symbolically written as

$$
\left[\frac{P}{Q}, \tau, \kappa, \mu\right] \rightarrow\left\{v_{l} \cdot(\mathbf{2 l}+\mathbf{1})\right\} \oplus\left[\frac{P^{\prime}}{Q^{\prime}}, q_{l}^{2 l+1} \tau, \pm \kappa, \pm \mu\right]
$$

Here the symbol $\left\{v_{l} \cdot(\mathbf{2 l}+\mathbf{1})\right\}$ denotes the string of the length $2 l+1$ with the parity $v_{l}$, $Q^{\prime}=Q-2 l+1, P^{\prime}=P-P^{\prime \prime}$ and $P^{\prime \prime}$ is the nearest integer to $(2 l+1) \eta$. If the string is open, i.e. $q_{l}^{2 l+1}=-1$ the numerators $P$ and $P^{\prime}$ have opposite parity (if $P$ is odd, $P^{\prime}$ is even and vice versa), while $P$ and $P^{\prime}$ have the same parity if the string is closed. The symbolic expression (29) means that Bethe roots corresponding to the state $\left[\frac{P}{Q}, \tau, \kappa, \mu\right]$ are given by 
the roots of the state $\left[\frac{P^{\prime}}{Q^{\prime}}, q_{l}^{2 l+1} \tau, \pm \kappa, \pm \mu\right]$ plus the roots of the string of the length $2 l+1$ with parity $v_{l}$.

The iterative procedure of the string's decomposition is identical to the hierarchical tree based on the spectral flow (Sec. III). Once the length of the highest string in a given band is the Hall conductance of the band, the string length is the rate of the spectral flow from the parent bands. Subtracting this string we arrive at the parent band of the generation given by the second term of the sequence of intermediate fractions (9)). Performing this procedure recursively, we obtain the entire set of possible lengths are the celebrated Takahashi-Suzuki numbers (10)) known from the $U_{q}\left(S l_{2}\right)$ integrable models.

Since lengths of strings are Hall conductances, the string content of a state can be read off from the hierarchical tree. The lengths of these strings are the Hall conductances of the bands on a branch (connecting these bands) that leads to the state. The string decomposition of the 15 bands of the example of the Sec. [1 $P / Q=4 / 15$ (11) is, as shown in Fig. 1 (only the lower half of the tree is shown):

$$
\begin{array}{ll}
J_{1}=(-1,-1,-1,-3,4,-4), & J_{9}=( \pm 2,1,-7,-4), \\
J_{2}=(-1,-1,-1,11), & J_{10}=( \pm 2,1,11), \\
J_{3}=(-1,-1,-1,7,-4), & J_{11}=( \pm 2,1,3,-4,-4), \\
J_{4}=(-1,-1,-4,4,-4), & J_{12}=(1,1,4,-4,-4), \\
J_{5}=( \pm 2,-1-3,4,-4), & J_{13}=(1,1,1,-7,-4), \\
J_{6}=( \pm 2,-1,11), & J_{14}=(1,1,1,11), \\
J_{7}=( \pm 2,-1,7,-4), & J_{15}=(1,1,1,3,-4,-4), \\
J_{8}=( \pm 2,-4,4,-4), &
\end{array}
$$

where in brackets we showed the lengths of strings and their parities as signs in front of lengths.

Fig. 2 shows the root patterns of the 7-th branch (counted from the bottom of the spectrum) $J_{7}$ of the tree from Fig. 1. We added also the root pattern of the 15-th level for the flux 9/34 which belongs to the same sequence. One can notice that the subsequent patterns of roots differ only by the string determined by hierarchy procedure. Although the topology of the hierarchical tree seems to be exact, its quantitative description in terms of strings is approximate. The true values of roots deviate from their bare value $x_{l}=1$ (see Fig. 2). Eq.(17) is approximate and valid only for long strings.

\section{GAP DISTRIBUTION AND BANDWIDTHS}

\section{A. Gaps}

A direct application of the string hypothesis is the gap distribution $\rho(D)$, i.e. the number of gaps with magnitude between $D$ and $D+d D$. This problem is essentially reduced to the scaling of the smallest gaps. Indeed, while moving along the tree new gaps appear, while older gaps become wider. There is no overlap between bands except at $E=0$ for even denominators of the flux (they touch) - all gaps are open [31].

Thus while moving from a generation $P^{\prime} / Q^{\prime}$ to generation $P / Q$, there will be $Q-Q^{\prime}$ new gaps. For typically Diophantine flux denominator grows fast with the generation number 
and $Q-Q^{\prime} \sim Q$. This means that the integral $\int_{D_{\min }}^{D_{\max }} \rho(D) d D=Q$ is saturated by the lower limit. If $\rho \sim D^{-\gamma}$, then $D_{\min }^{-\gamma+1} \sim Q$.

To find the size of the minimal gap, let us consider $P$-even and $\tau=-1$. Then the Bethe equations give edges of gaps. Let us consider an arbitrary gap in this generation and trace the genealogy of lower and upper edges of the gap along two paths $J_{-}$and $J_{+}$of the tree, until we find the nearest common ancestor at the generation with denominator $Q^{\prime}$. The minimal gap would correspond to the shortest paths $J_{ \pm}$, i.e. when $Q^{\prime}$ is the denominator of the generation parent to $P / Q$. The string decompositions of the two edges have a common part consisting of strings of length smaller than $Q^{\prime}$ (they correspond to the common path from the origin of the tree to the parent generation $Q^{\prime}$ ) and different strings with length bigger than $Q^{\prime}$ belonging to the paths $J_{ \pm}$. Then according to (16), the width of the gap is the difference between energies of strings with length greater than $Q^{\prime}$ along the path $J_{-}$and the energies of strings along $J_{+}$. The contribution to the energy $\varepsilon_{l}$ from a string with a spin $l$ which connects the center of the band $(\tau=+1)$ with the edge of the band $(\tau=-1)$ is

$$
\varepsilon_{l}=i\left(q-q^{-1}\right) 2\left(\sum_{k=-l}^{l} x_{l} v_{l} q_{l}^{k}-\frac{2 i}{q^{1 / 2}-q^{-1 / 2}}\right) \approx \frac{4 \pi}{Q(2 l+1)} \frac{\cos ^{2}\left(\frac{\pi}{2} \frac{P}{Q}\right)}{\sin \left(\frac{\pi}{2} \frac{P}{Q}\right)} \sim \frac{1}{Q^{2}},
$$

where we used the fact that for a typical Diophantine flux $\eta$ the length of the largest string $2 l+1$ scales as $Q$ along the sequence of rational approximants. This gives us an estimate for the gap $E_{l} \sim 1 / Q^{2}$. The difference of energies of strings is of the same order. This gives $D_{\min } \sim 1 / Q^{2}$ and the gap distribution function $\rho(\mathcal{D}) \sim \mathcal{D}^{-3 / 2}$. This result has been obtained numerically in Ref. [32].

\section{B. Bands. Stating a Problem}

The bandwidths are a more subtle matter. Let us take $k$-th band belonging to some generation with $P$-even at $\tau=-1$. Then $E_{k}$ is the edge of this band. We can take one step back along the hierarchical tree. We find that the parent band $k^{\prime}$ is of generation $\eta^{\prime}=P^{\prime} / Q^{\prime}$ with $P^{\prime}$-odd and $\tau=+1$. The rational points at this generation give centers of bands. If we had started from the band $Q+1-k$ (symmetric to the $k$-th band) we would find the band $Q^{\prime}+1-k^{\prime}$ as its parent band. The roots of symmetric bands of generation $\eta^{\prime}$ are related by symmetry $z_{i} \rightarrow-z_{i}$ (see Sec. [X]). Thus the string content of two paths leading to the bottom and the top edges of the band differ only by the highest string of the same length $l$ and the same parity $v_{l}$. If we assume that adding a string does not change the centers of already existing strings (the noninteracting strings approximation), then the bandwidth is given just by twice the energy of the highest string (31). This gives an estimate of the bandwidth $W \sim \frac{1}{Q^{2}}$. Corrections to this crude estimate come from many sources (roots get some small corrections when the new string is added, every root has "finite size" corrections) and these are of the order $Q^{-2}$ as well.

It has been shown by Thouless [33] that the sum of all bandwidths scales as $Q^{-1}$ (moreover $\lim _{Q \rightarrow \infty} Q \sum_{k} W_{k}=$ const $=9.32 \ldots$ ). If all bands scale similarly (fractal), then the scaling dimension $Q^{-2}$ would fit the scaling of the total bandwidth. We call this the mean dimension. In reality, bandwidths scale differently (multifractal). Their anomalous dimensions 
$\epsilon_{J}$ depend on the path $J$ of the hierarchical tree that the band belongs to: $W(J) \sim Q^{-2+\epsilon_{J}}$, where $Q$ is the denominator of the flux running along the path. According to ref. [34 for $\eta=\frac{\sqrt{5}-1}{2}$ exponents $\epsilon_{J}$ vary between 0.171 and -0.374 , where the positive anomalous dimension corresponds to a path in the center of the tree, while the negative one belongs to the path along the lowest bands.

A distribution of anomalous dimensions of the bandwidths, can be described by the function: $F(\beta)=-\lim _{Q \rightarrow \infty} \frac{\log \sum_{k=1}^{Q} W_{k}^{\beta}}{\log Q}$ Here is a limited list of what we do know about this function. The function $F(\beta)$ is convex and has linear asymptotes $F(\beta)=\frac{\beta}{\epsilon_{\min }}$ for $\beta \rightarrow-\infty$ and $F(\beta)=\frac{\beta}{\epsilon_{\max }}$ for $\beta \rightarrow \infty$. A deviation of $F(\beta)$ from a straight line (fractal) indicates multifractality. The point $\beta=D_{\mathrm{H}}$ where $F\left(D_{\mathrm{H}}\right)=0$ is known as the "Hausdorff dimension" and the slope of $F$ at this point, gives the most probable scaling dimension $\epsilon_{0}=F^{\prime}\left(D_{\mathrm{H}}\right)$. The scaling of the total bandwidth 10,33 gives $F(-1)=1$. Obviously, $F(0)=-1$. We have plotted $F(\beta)$ for the case where the flux is equal to the golden mean in Fig. 6 .

If $F(\beta)$ were a straight line, $D_{\mathrm{H}}$ would be $\frac{1}{2}$ and $\epsilon_{0}=2$. It would mean that each scaling dimension $\epsilon(J)$ is equal to the mean field value 2. From Fig. 6 we can see that $F(\beta)$ is indeed close to a straight line. The Hausdorff dimension $D_{\mathrm{H}}=0.49792 \pm 4 \times 10^{-5}$ and the most probable scaling dimension $\epsilon_{0}=1.9996 \pm 1 \times 10^{-4}$ are numerically close to $\frac{1}{2}$ and 2 respectively. Anomalous dimensions are small but code the most interesting features of the problem.

The topological classification of solutions to the Bethe equations by means of strings alone gives the mean-field dimension of bandwidths and absolute values of bandwidths with high accuracy of the order of $1 / Q^{2}$. Analytical determination of anomalous dimensions goes beyond just the topological classification and is perhaps the most challenging problem in the field. We do not attempt to solve it in this paper.

\section{NUMERICAL EVIDENCE FOR THE STRING-HIERARCHY HYPOTHESIS}

In this Section we present a fragment of an extensive numerical analysis we have performed in order to confirm the string-hierarchy hypothesis of the Sec. IV. We choose the most "irrational" flux (golden mean) $\eta=\frac{\sqrt{5}-1}{2}=[1 ; 1,1,1,1 \ldots]$. This is the only case where intermediate fractions are the same as convergents

$$
\frac{P_{i}}{Q_{i}}=\frac{F_{i-1}}{F_{i}}=\frac{1}{1}, \frac{1}{2}, \frac{2}{3}, \frac{3}{5}, \frac{5}{8}, \frac{8}{13}, \ldots,
$$

where $F_{i}$ are Fibonacci numbers as well. They give the fluxes of generations. The set of Hall conductances $=$ Takahashi-Suzuki numbers $=$ allowed lengths of strings and are all Fibonacci numbers:

$$
2 l_{k}+1=Q_{k-1}=F_{k-1}=1,1,2,3,5,8, \ldots
$$

As an illustration let us consider the bottom edge of the lowest energy band of the generation $\frac{34}{55}$. According to the string hierarchy hypothesis the length of the largest string is 21 and then iteratively one obtains that the string content consists of strings of lengths $F_{3 k+1}=1,5,21$ for $k=0,1,2$ and can be written as $(1,1,5,5,21,21)$. All string parities 


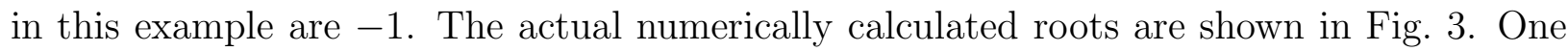
notices immediately that the roots organize themselves into distinct groups whose members have roughly the same radius: two groups with $1 \operatorname{root}(+)$, two with $5 \operatorname{roots}(\times)$, and two groups with $21 \operatorname{roots}(*)$.

The spins of strings and their parities are topological characteristics of the state. In contrast to these properties, the centers of strings are not topological and become close to 1 only for large spin strings. For our example they are $x=2.10,(2.10)^{-1}, 1.23,(1.23)^{-1}$, $0.81,(0.81)^{-1}, 1.05,(1.05)^{-1}$. We have found numerically that the centers of large strings are $x_{F_{3 n+1}} \approx 1+\frac{\xi}{F_{3 n+1}}$ with $\xi=1.14$.

Another example, Fig. 田, illustrates the hierarchy. It shows how 12 roots of the band edges of the generations $\frac{8}{13}$ can be approximately obtained from 4 roots of the centers of the bands for the parent generation $\frac{3}{5}$ and 7 roots of generation $\frac{5}{8}(\diamond)$ by the addition of the strings of the lengths 8 and $5(\square)$ respectively.

The string decomposition gives an asymptotic form of the wave function (18). In particular the wave function of the bottom edge of the lowest band of the generation $F_{3 n-1} / F_{3 n}$ is

$$
\psi^{\left\{F_{3 n-2}, \ldots\right\}}(z) \approx \prod_{i=k}^{n-1} \prod_{m=1}^{F_{3 i+1}}\left(z-x_{F_{3 i+1}} q_{3 i+1}^{-\frac{F_{3 i+1}+1}{2}+m}\right)\left(z-x_{F_{3 i+1}}^{-1} q_{3 i+1}^{-\frac{F_{3 i+1}+1}{2}+m}\right) \psi^{\left\{F_{3 k-2}, \ldots\right\}}
$$

where $q_{j}=e^{i \pi \frac{F_{j-1}}{F_{j}}}$. This formula is asymptotically exact at $k \rightarrow \infty$, but also works very well even for $k=0, \psi^{\{0\}}=1$ and $\psi^{\left\{F_{3 k-2}, \ldots\right\}}$ is the wave function of the generation $F_{3 k-1} / F_{3 k}$.

First of all let us check that the formula (34) is qualitatively correct and gives the same root pattern as the actual roots of the Bethe Ansatz equations. In Fig. 3 we have presented, in addition to the numerically calculated roots corresponding to the ground state of equation (12) for the flux $\eta=34 / 55$, also the roots given by (34). It can be seen that the roots produced by (34) are in quite good agreement with the numerical results. Actually one can check that the numerical accuracy with which formula (34) gives roots is $\sim 1 / l^{2}$ for each root of the string of the length $l$. Now let us calculate the overlap between this wave function (34) and the direct numerical solution of the eq.(44).

The result is tabulated in the following table, where the overlap between the wave function obtained from the exact (numerically obtained) roots $\psi_{\text {Exact }}$ and ansatz wave function of different order $k: \psi_{\text {Ansatz }}^{\{k\}}$ is shown. The ansatz wave function of the order $k$ is given by eq.(34). It is "made" out of real (numerical) and ansatz roots. The order $k$ of the ansatz function tells how many of the longest strings are replaced by ansatz ones. $k=0$ corresponds to pure ansatz wave function. The bigger $k$ the more short strings are replaced by ansatz strings. The positions of centers of the ansatz strings are taken to be $x_{F_{3 i+1}}=1+\xi / F_{3 i+1}$ with the only fitting parameter $\xi$ which is chosen to be $\xi=1.14$.

\begin{tabular}{|c|c|c|c|c|c|c|}
\hline$k$ & 0 & 1 & 2 & 3 & 4 & 5 \\
\hline$\left\langle\psi_{\text {Ansatz }}^{\{k\}} \mid \psi_{\text {Exact }}\right\rangle$ & 0.99475 & 0.99464 & 0.99657 & 0.99669 & 0.999435 & 1.000000 \\
\hline
\end{tabular}

The last column of this table reflects trivially the normalization of wave function. One can notice that for $k=4$ when two strings of the length 377 are replaced by the ansatz roots the overlap between ansatz wave function and exact one is 0.9994 which is very close to 1 . 
Actually for this case even the purely ansatz wave function (with all roots produced from (34)) has a good overlap: 0.995 with the exact one.

A more subtle check of the high degree of similarity between the exact and ansatz wave function is the distribution of scaling exponents of moments of the wave function, which is often used to characterize the multifractal properties of the set generated by of values of $\psi_{n}$ [35]. This distribution is given by the Legendre transformation

$$
\alpha=\frac{1}{F^{\prime}(\beta)}, \quad f(\alpha)=\beta-\frac{F(\beta)}{F^{\prime}(\beta)} .
$$

of the function

$$
F(\beta)=\lim _{Q \rightarrow \infty} \frac{\log \sum_{n=1}^{Q}\left|\psi_{n}\right|^{\beta}}{\log Q} .
$$

An extended support of $f(\alpha)$ gives some measure of multifractality.

In Fig. 5 we have plotted $f(\alpha)$ for $\psi_{\text {Ansatz }}$ from eq. (34) and $\psi_{\text {Exact }}$ from the exact roots, calculated numerically for a particular value of the flux, $\eta=610 / 987$. By representing the data in this way we have neglected taking the limit in eq. (36). For this value of the flux this is justifiable by the fact that when increasing the denominator of the flux beyond 987, $F(\beta)$ does not vary visibly. The figure shows that the ansatz approaches the exact $f(\alpha)$ remarkably well, with just one free parameter $\xi \approx 1.14$.

Concerning the support of $f(\alpha), \alpha_{\min }$ is approximately 0.32 for the ansatz and 0.31 for the exact wave function, whereas $\alpha_{\max }$ is approx. 3.5 for both ansatz and exact wave function within margin of error.

\section{CONCLUSIONS}

The Azbel-Hofstadter problem as a typical quasiperiodic system generates a complex spectrum. Similar complex spectra were found in a number of dynamical systems. Since the empirical observations of Hofstadter [2], evidence has been mounting that these spectra are regular and universal rather than erratic or "chaotic". They have a deterministic hierarchical structure. In this paper we have shown that the topology of the strange sets, generated in the problem is determined by the Chern numbers of the spectral curve i.e. by the Hall conductances. Even more so - at every finite step of the hierarchy the spectrum is integrable. We have found an anticipated match between Hall conductances and dimensions of representations of the quantum group $U_{q}\left(s l_{2}\right)$. The latter are Takahashi-Suzuki numbers or the lengths of the strings of the Bethe Ansatz solution. This correspondence suggests a natural hierarchical tree, which, we believe, is relevant for general quasiperiodic systems.

In the Bethe Ansatz language, each state is characterized by a particular string content. Proceeding along the tree toward the incommensurate limit corresponds to addition of strings. This picture is somewhat reminiscent of the discrete renormalization group approach [36].

We were concentrated on the topological aspect of the string hypothesis. It alone gives 
- The explicit asymptotically exact form of some wave functions for irrational flux. Multi-fractal properties of these functions, although not exact, are in good agreement with numerical results.

- The scaling exponent $3 / 2$ of the gap distribution.

- The most probable scaling dimension $1 / 2$ of the bandwidths.

However, the major ends of the strings are loose. The string hypothesis solves the Bethe Ansatz equations with an accuracy $\mathcal{O}\left(Q^{-2}\right)$, i.e. is asymptotically exact in the incommensurate limit $Q \rightarrow \infty$. However, the most interesting quantitative characteristics of the spectrum are actually in the finite size corrections of the order of $\mathcal{O}\left(Q^{-2}\right)$ to the bare value of strings. Among them are the anomalous dimensions of the spectrum. We believe that it is possible to find them analytically via a more detailed study of the Bethe Ansatz equations, beyond just the analysis of singularities. This is a technically involved but a fascinating problem. The ultimate solution of the problem, however, would be through the application of conformal field theory approach, which has been proven to be effective for finding the finite size corrections of integrable systems, without the actual solution of the Bethe Ansatz.

\section{ACKNOWLEDGMENTS}

We would like to thank J. Bellissard, who suggested that the noninteracting strings lead to the scaling exponent $-3 / 2$ for the gap distribution function and pointed out the Ref. [32], and Y. Hatsugai for inspiring numerics during the initial stages of this project. We acknowledge useful discussions with Y. Avron, G. Huber, S. Jitomirskaya, M. Kohmoto, Y. Last, R. Seiler and A. Zabrodin. AGA was supported by MRSEC NSF Grant DMR 9400379. PBW was supported under NSF Grant DMR 9509533.

\section{APPENDIX: BETHE-ANSATZ EQUATIONS FROM HOFSTADTER MODEL.}

In this section we (re)derive the Bethe Ansatz equations for the Azbel-Hofstadter problem with an anisotropy parameter $\lambda[28,29]$.

The Hamiltonian of a particle on a lattice in a magnetic field can be written as

$$
H=T_{x}+T_{x}^{-1}+\lambda\left(T_{y}+T_{y}^{-1}\right),
$$

where the translations operators on a square lattice in a magnetic field $2 \pi \eta$ per plaquette form a Weyl pair

$$
T_{x} T_{y}=q^{2} T_{y} T_{x} .
$$

The representation $T_{x} f_{n}=f_{n+1}, T_{y} f_{n}=q^{2 n} e^{i k_{y}} f_{n}$ with $q=e^{i \pi \eta}$ gives the Harper equation (11). If the flux through a plaquette of the square lattice is a rational fraction of the flux quantum $\eta=P / Q$ then the operator $T_{x}^{Q}$ commutes with the Hamiltonian (37) and there are finite dimensional representations characterized by two momenta $k_{x}, k_{y}$ so that 


$$
T_{x}^{Q}=e^{i Q k_{x}}, T_{y}^{Q}=e^{i Q k_{y}} .
$$

The non-equivalent representations are given by restricting $0 \leq k_{x}<2 \pi / Q$ and $0 \leq k_{y}<2 \pi$ and Schrödinger equation becomes:

$$
E \psi_{n}=e^{i k_{x}} \psi_{n+1}+e^{-i k_{x}} \psi_{n-1}+2 \lambda \cos \left(2 \pi \eta n+k_{y}\right)
$$

with periodic boundary conditions $\psi_{n+Q}=\psi_{n}$. The Chambers relation [37 for the problem (40) says that the energy spectrum is obtained as the solutions of an algebraic equation $\operatorname{Pol}(E)=\Lambda\left(k_{x}, k_{y}\right)$ where $\operatorname{Pol}(E)=E^{Q}+\ldots$ is a polynomial of $Q$-th degree which coefficients do not depend on $k_{x}$ and $k_{y}$. The only dependence of energy on $k_{x}$ and $k_{y}$ comes from free term of algebraic equation via the combination:

$$
\Lambda\left(k_{x}, k_{y}\right)=2 \cos Q k_{x}+2 \lambda^{Q} \cos Q k_{y} .
$$

Therefore the edges of the energy bands are given by extrema of $\Lambda$ which assumes a minimum/maximum given by $\Lambda= \pm 2\left(1+\lambda^{Q}\right)$.

Below we use another representation (the modified chiral gauge):

$$
T_{x}=U V \frac{U+a}{U+b}, \quad T_{y}=V U^{-1} \frac{U+a}{U+b}, \quad U V=q V U .
$$

In this representation for the choice

$$
\begin{aligned}
& a=-i \kappa \lambda^{-\frac{1}{2}} q^{-\frac{1}{2}} \\
& b=-i \tau \kappa \lambda^{\frac{1}{2}} q^{-\frac{1}{2}}
\end{aligned}
$$

where $\tau, \kappa= \pm 1$ the Hamiltonian (37) becomes

$$
\begin{aligned}
H= & q V U^{-1}\left(U-i \kappa \lambda^{-\frac{1}{2}} q^{-\frac{1}{2}}\right)\left(U+i \tau \kappa \lambda^{\frac{1}{2}} q^{-\frac{1}{2}}\right) \\
& +\lambda q^{-1} V^{-1} U^{-1}\left(U+i \kappa \lambda^{-\frac{1}{2}} q^{\frac{1}{2}}\right)\left(U-i \tau \kappa \lambda^{\frac{1}{2}} q^{\frac{1}{2}}\right) .
\end{aligned}
$$

Let us choose the following representation of operators $U$ and $V$ :

$$
(U \psi)_{n}=e^{i p_{-}} q^{-n} \psi_{n}, \quad(V \psi)_{n}=-i \tau \kappa e^{i p_{+}} \lambda^{\frac{1}{2}} \psi_{n+1} .
$$

Then the Schrödinger equation corresponding to (43) becomes

$$
\begin{aligned}
-\kappa q^{n} e^{-i p_{-}} \lambda^{-\frac{1}{2}} E \psi_{n} & =i q e^{i p_{+}}\left(q^{n} e^{-i p_{-}}-i \tau \kappa \lambda^{-\frac{1}{2}} q^{-\frac{1}{2}}\right)\left(q^{n} e^{-i p_{-}}+i \kappa \lambda^{\frac{1}{2}} q^{-\frac{1}{2}}\right) \psi_{n+1} \\
& -i q^{-1} e^{-i p_{+}}\left(q^{n} e^{-i p_{-}}+i \tau \kappa \lambda^{-\frac{1}{2}} q^{\frac{1}{2}}\right)\left(q^{n} e^{-i p_{-}}-i \kappa \lambda^{\frac{1}{2}} q^{\frac{1}{2}}\right) \psi_{n-1}
\end{aligned}
$$

The equation (44) has periodic coefficients and one can require that $\psi_{n+Q}=\psi_{n}\left(\psi_{n+2 Q}=\psi_{n}\right)$ for $P$-even ( $P$-odd). Then the solutions will be labeled by $0 \leq p_{+}<2 \pi / Q$ for $P$-even and by $0 \leq p_{+}<\pi / Q$ for $P$-odd.

Let us now extend the periodic function $\psi_{n}$ to the entire complex plane by writing $\psi_{n}=\left.\Psi(z)\right|_{z=-e^{-i p_{-}} q^{n}}$. We obtain: 


$$
\begin{aligned}
\kappa \lambda^{-\frac{1}{2}} z E \Psi(z) & =i q e^{i p_{+}}\left(z+i \tau \kappa \lambda^{-\frac{1}{2}} q^{-\frac{1}{2}}\right)\left(z-i \kappa \lambda^{\frac{1}{2}} q^{-\frac{1}{2}}\right) \Psi(q z) \\
& -i q^{-1} e^{-i p_{+}}\left(z-i \tau \kappa \lambda^{-\frac{1}{2}} q^{\frac{1}{2}}\right)\left(z+i \kappa \lambda^{\frac{1}{2}} q^{\frac{1}{2}}\right) \Psi\left(q^{-1} z\right) .
\end{aligned}
$$

If $e^{2 i p_{+}}=1$, the eq. (45) has $Q$ polynomial solution of the order $Q-1$. Below we denote $\mu=e^{i p_{+}}= \pm 1$. The change $\mu \rightarrow-\mu$ changes the sign of energy corresponding to given polynomial solution of (45). Let us parameterize the polynomial solutions by its roots $z_{i}$ :

$$
\Psi(z)=\prod_{i=1}^{Q-1}\left(z-z_{i}\right)
$$

Evaluating eq. (45) at one of the roots $z_{i}$, we obtain the Bethe Ansatz equations:

$$
q^{Q} \prod_{j=1}^{Q-1} \frac{q z_{i}-z_{j}}{z_{i}-q z_{j}}=\frac{\left(z_{i}-i \tau \kappa q^{\frac{1}{2}} \lambda^{-\frac{1}{2}}\right)\left(z_{i}+i \kappa q^{\frac{1}{2}} \lambda^{\frac{1}{2}}\right)}{\left(q^{\frac{1}{2}} z_{i}+i \tau \kappa \lambda^{-\frac{1}{2}}\right)\left(q^{\frac{1}{2}} z_{i}-i \kappa \lambda^{\frac{1}{2}}\right)}
$$

These equations have $Q$ independent solutions. Each of them consists of $Q-1$ complex numbers $z_{k}, k=1, \ldots, Q-1$. Comparing the coefficients of $z^{Q}$ and $z$ on the left and right sides of equation (45) one finds the energy:

$$
E=i \mu q^{Q}\left(q-q^{-1}\right) \lambda^{1 / 2}\left[\kappa \sum_{i=1}^{Q-1} z_{i}-\frac{i}{q^{1 / 2}-q^{-1 / 2}}\left(\lambda^{1 / 2}-\tau \lambda^{-1 / 2}\right)\right]
$$

or

$$
E=i \mu\left(q-q^{-1}\right) \lambda^{1 / 2}\left[\kappa \sum_{i=1}^{Q-1}-\tau z_{i}^{-1}-\frac{i}{q^{1 / 2}-q^{-1 / 2}}\left(\lambda^{1 / 2}-\tau \lambda^{-1 / 2}\right)\right] .
$$

Combining these two formula we have a more convenient form of the energy

$$
E=i \mu \frac{1}{2} q^{Q}\left(q-q^{-1}\right) \lambda^{1 / 2}\left[\kappa \sum_{i=1}^{Q-1}\left(z_{i}-q^{-Q} \tau z_{i}^{-1}\right)-i \frac{1-q^{-Q}}{q^{1 / 2}-q^{-1 / 2}}\left(\lambda^{1 / 2}-\tau \lambda^{-1 / 2}\right)\right]
$$

The Bethe equations (47) possess some discrete symmetries: if the set $\left\{z_{i}\right\}$ is a solution with anisotropy $\lambda$ and energy $E$ then $\left\{z_{i}^{*}\right\}$ is also a solution with the same energy and $\left\{-\tau z_{i}^{-1}\right\}$ is a solution with the energy $q^{Q} E$. In its turn the set $\left\{z_{i}^{-1}\right\}$ is a solution of the problem with $\lambda^{-1}$, its energy is $-\tau q^{Q} E$. Finally, there is a trivial symmetry of equations $z_{i}, \kappa \rightarrow-z_{i},-\kappa$ which does not change the energy.

After some algebra one finds a relation between the wave function of the Harper equation (40) and polynomial (46).

(i) In the case of $q^{Q}=-1(P$-odd $)$ and in the middle of bands the relation is

$$
\psi_{n}=(-1)^{n} q^{-n^{2}+2 n} \sum_{m=0}^{2 Q-1} \prod_{j=0}^{m-1}\left(e^{i k_{y}} q^{2 n+\frac{1}{2}} \frac{\lambda^{\frac{1}{2}}-\tau \kappa e^{-i \frac{k_{x}+k_{y}}{2}} q^{-j-\frac{1}{2}}}{\lambda^{\frac{1}{2}}-\kappa e^{i \frac{k_{x}+k_{y}}{2}} q^{j+\frac{1}{2}}}\right) \Psi\left(-i e^{i \frac{k_{x}+k_{y}}{2}} q^{m}\right),
$$

where $k_{x}, k_{y}$ satisfy the condition $\Lambda\left(k_{x}, k_{y}\right)=0$. 
(ii) In the case of $q^{Q}=+1$ (P-even, $Q$-odd) and $\Lambda=2 \mu(-1)^{\frac{P}{2}}\left(\lambda^{Q}-\tau\right)$ the relation is

$$
\psi_{n}=q^{-n^{2}} \sum_{m=0}^{Q-1} \prod_{j=0}^{m-1}\left(e^{i k_{y}} q^{2 n+\frac{1}{2}} \frac{\lambda^{\frac{1}{2}}-i \tau \kappa e^{-i \frac{k_{x}+k_{y}}{2}} q^{-j-\frac{1}{2}}}{\lambda^{\frac{1}{2}}+i \kappa e^{i \frac{k_{x}+k_{y}}{2}} q^{j+\frac{1}{2}}}\right) \Psi\left(-e^{i \frac{k_{x}+k_{y}}{2}} q^{m}\right) .
$$

If $\tau=+1$ it corresponds to the middle of bands and the rational points $k_{x}, k_{y}$ given by the equation $\frac{\cos \frac{Q}{2}\left(k_{x}+\pi \frac{P}{2 Q}\right)}{\sin \frac{Q}{2}\left(k_{y}+\pi \frac{P}{2 Q}\right)}=\kappa(-1)^{\frac{Q-1}{2}} \lambda^{\frac{Q}{2}}$. If $\tau=-1, \Lambda=2 \mu(-1)^{\frac{P}{2}}\left(\lambda^{Q}+1\right)$ the relation corresponds to the points at the edges of bands $k_{x}=\frac{\pi}{Q} \frac{1-(-1)^{\frac{P}{2}}}{2}$ and $k_{y}=\frac{\pi}{Q}\left(\frac{1-(-1)^{\frac{P}{2}}}{2}+2 l\right)$ for $l=0,1, \ldots, Q-1$. If counted from the bottom of the spectrum, these edges are ordered as bottom-top-bottom. . if $\mu(-1)^{\frac{P}{2}}=-1$ and top-bottom-top. . if $\mu(-1)^{\frac{P}{2}}=+1$. 


\section{REFERENCES}

[1] M.Ya. Azbel, Zh. Eksp. Teor. Fiz. 46, 929 (1964)

[ Sov. Phys. JETP, 19, 634-645 (1964)]

Energy spectrum of a conduction electron in a magnetic field.

[2] D.R. Hofstadter, Phys. Rev. B 14, 2239-2249 (1976)

Energy levels and wave functions of Bloch electrons in a rational or irrational magnetic field.

[3] S. Aubry, G. Adnré, Ann. Israel Phys. Soc. 3, 133-164 (1980)

Analyticity breaking and Anderson localization in incommensurate lattices.

[4] Ya.G. Sinai, J. Stat. Phys. 46, 861-909 (1987)

Anderson localization for one-dimensional difference Schrödinger operator with quasiperiodic potential.

[5] Y. Last, Proc. XI Intern. Congress of Math. Phys. (Paris 1994)

Almost everything about the almost Mathieu operator I.

S. Jitomirskaya, ibid.

Almost everything about the almost Mathieu operator II.

[6] D.J. Thouless, Ch. 4 in "The Quantum Hall Effect", ed. R.E. Prange, S.M. Girvin, Sringer-Verlag (1987)

Topological considerations.

[7] K. Iguchi, Int. J. Mod. Phys. B11, 2405-2423 (1997)

Tight-binding model for DNA double chains: Metal-insulator transitions due to the formation of a double strand of DNA.

[8] Ch. Kreft, R. Seiler, Sfb 288 Preprint No.209

Models of Hofstadter type.

[9] J. Bellissard, B. Simon, J. Funct. Anal. 48, 408-419 (1982)

Cantor spectrum for the almost Mathieu equation.

[10] D.J. Thouless, Phys. Rev. B 28, 4272-4276 (1983)

Bandwidths for a quasi-periodic tight-binding model.

[11] J. Avron, P. van Mouche, B. Simon, Commun. Math. Phys. 132, 103-118 (1990)

On the measure of the spectrum for the almost Mathieu operator.

[12] Y. Last, Commun. Math. Phys. 164, 421-432 (1994)

Zero measure spectrum for the almost Mathieu operator.

[13] B. Simon, Adv. Appl. Math. 3, 463-490 (1982)

Almost periodic Schrödinger operators: A review.

[14] C. Tang, M. Kohmoto, Phys. Rev. B 34, 2041 (1986)

Global scaling properties of the spectrum for a quasiperiodic Schrödinger equation.

[15] P.B. Wiegmann and A.Zabrodin, Nucl. Phys. B 422, 495 (1994)

Bethe-Ansatz Approach to the Azbel-Hofstadter Problem. Quantum Group and Magnetic Translations.

idem Phys. Rev. Lett. 72, 1890 (1994)

Bethe Ansatz for Bloch Particles in Magnetic Field

idem Mod. Phys. Lett. B 8, 311 (1994)

Quantum group and magnetic translations. Bethe ansatz solution for the Harper's equation. 
[16] Y. Hatsugai, M. Kohmoto, Y.S. Wu, Phys. Rev. Lett. 73, 1134 (1994)

Explicit Solutions to the Bethe-Ansatz Equations for Bloch Electrons in a Magnetic Field. idem Phys. Rev. B 53, 9697 (1996) Quantum Group, Bethe Ansatz Equations and Bloch Electron in a Magnetic Field.

[17] Minoru Takahashi, Masuo Suzuki, Progr. Theor. Phys. 48, 2187 (1972) One-dimensional anisotropic Heisenberg model at finite temperatures.

[18] A number of different hierarchical trees has been discussed in Refs. [2], [19], [20].

[19] R.B. Stinchcombe, S.C. Bell, J. Phys. A: Math. Gen. 20, L739-L744 (1987) Hierarchical band clustering and fractal spectra in incommensurate systems.

[20] Greg Huber, Preben Alstrom, H. Eugene Stanley, J. Phys. A: Math. Gen. 22, L279 (1989) Number of scaling factors in incommensurate systems.

[21] I.M. Vinogradov, p.15, problem 4(b) in Pergamon press (1961) An introduction to the theory of numbers.

[22] P. Streda, J. Phys. C: Solid State Phys., 15, L717-L721 (1982) Theory of quantized Hall conductivity in two dimensions.

[23] B.A. Venkov, WN publishers, 1970 Elementary number theory.

[24] D.J. Thouless, M. Kohmoto, M.P. Nightingale, M. den Nijs, Phys. Rev. Lett. 49, 405 (1982) Quantized Hall conductance in a two-dimensional periodic potential.

[25] I. Dana, Y. Avron, J. Zak, J. Phys. C: Solid State Phys. 18, L679-L683 (1985) Quantized Hall conductance in a perfect crystal.

[26] For rigorous definition of best approximants and for correct formulation of the theorem that all best approximants are given by intermediate fractions see [23].

[27] L. Mezincescu, R. I. Nepomechie, Phys. Lett., B246, 412-416 (1990) Unitarity and irrationality for the quantum algebra $U_{q}[S U(2)]$.

[28] L.D. Faddeev, R.M. Kashaev, Comm. Math. Phys., 169, 181 (1995) Generalized Bethe Ansatz Equations for Hofstadter Problem.

[29] A. Zabrodin in Proc. of the third Intern. School on Theor. Physics, Poznan, Poland, Sept. 1994: "Symmetry and Structural Properties of Condensed Matter", edited by T. Lulek, W. Florek and S. Walcerz (World Scientific, Singapore-River Edge, NJ), 1995.

[30] C.M. Yung, M.T. Batchelor, Nucl. Phys. B446, 461-484 (1995) Exact solution for the spin-s XXZ quantum chain with non-diagonal twists

[31] P. v. Mouche, Commun. Math. Phys. 122, 23-34 (1989) The coexistence problem for the discrete Mathieu operator.

[32] T. Geisel, R. Ketzmerick, G. Petschel, Phys. Rev. Lett. 66, 1651-1654 (1991) New class of level statistics in quantum systems with unbounded diffusion.

[33] D.J. Thouless, Commun. Math. Phys. 127, 187-193 (1990) Scaling for the discrete Mathieu equation.

[34] H. Hiramoto, M. Kohmoto, Phys. Rev. B40, 8225 (1989) Scaling analysis of quasi-periodic systems: Generalized Harper model.

[35] T.C. Halsey, M.H. Jensen, L.P. Kadanoff, I. Procaccia, B.I. Shraiman, Phys. Rev. A 33, $1141(1986)$ 
Fractal measures and their singularities: The characterization of strange sets.

[36] S. Ostlund, R. Pandit, Phys. Rev. B 29, 1394-1414 (1984)

Renormalization-group analysis of the discrete quasiperiodic Schrödinger equation.

[37] W.G. Chambers, Phys. Rev. A 140, 135-143 (1965)

Linear network model for magnetic breakdown in two dimensions. 


\section{FIGURES}

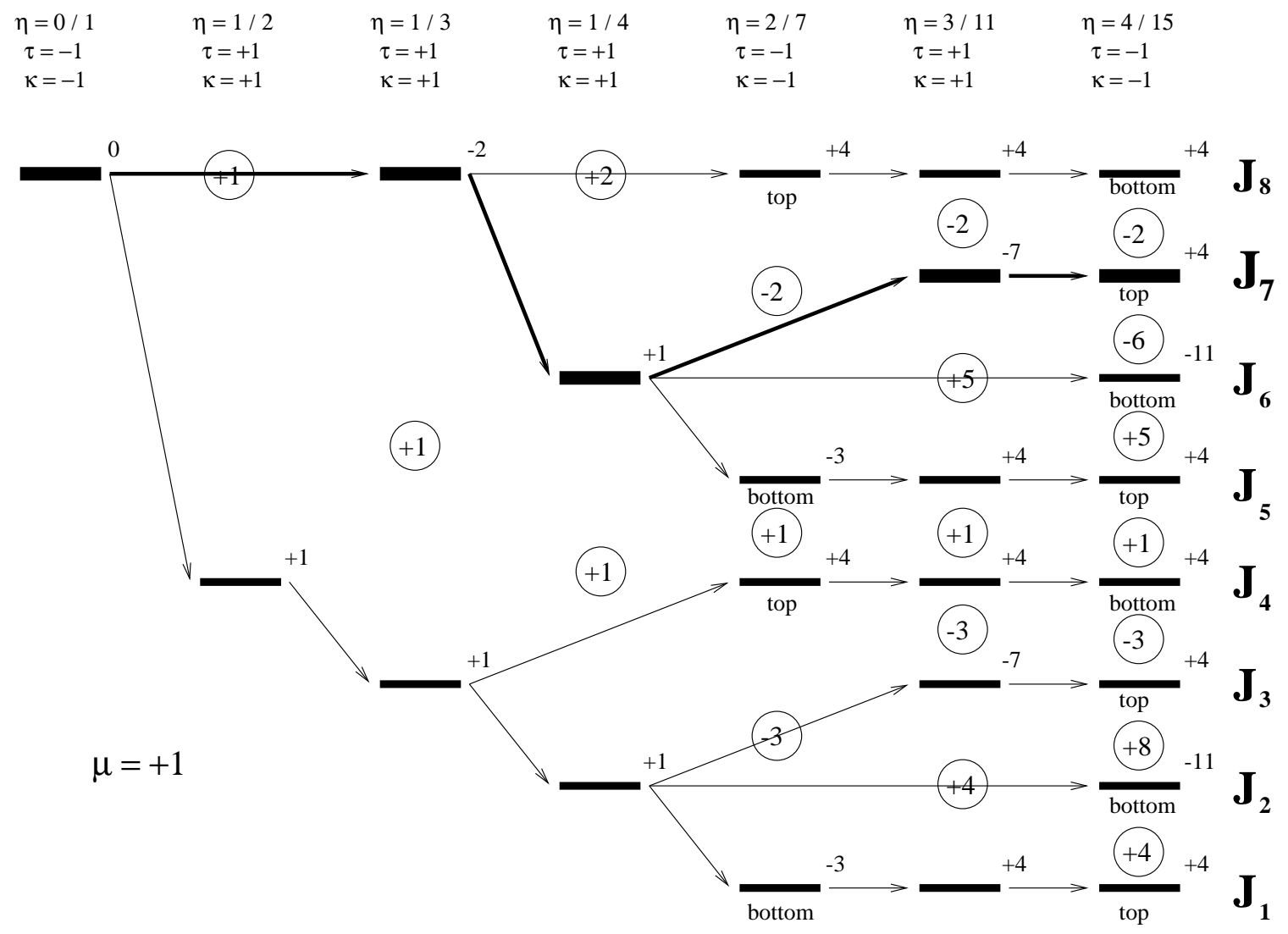

FIG. 1. The lower half of the hierarchical tree for the flux $\eta=4 / 15$ is shown. Nodes which lie on a vertical slice of the tree are bands of a generation ordered with respect to their energies. The numbers in the figure are Hall conductances of bands and gaps. The Hall conductances in gaps are shown as encircled numbers. "Top" and "bottom" are the edges of a band. For all states shown $\mu=+1$. Root patterns of the highlighted branch $J_{7}$ of the tree are shown in Fig. 2. 


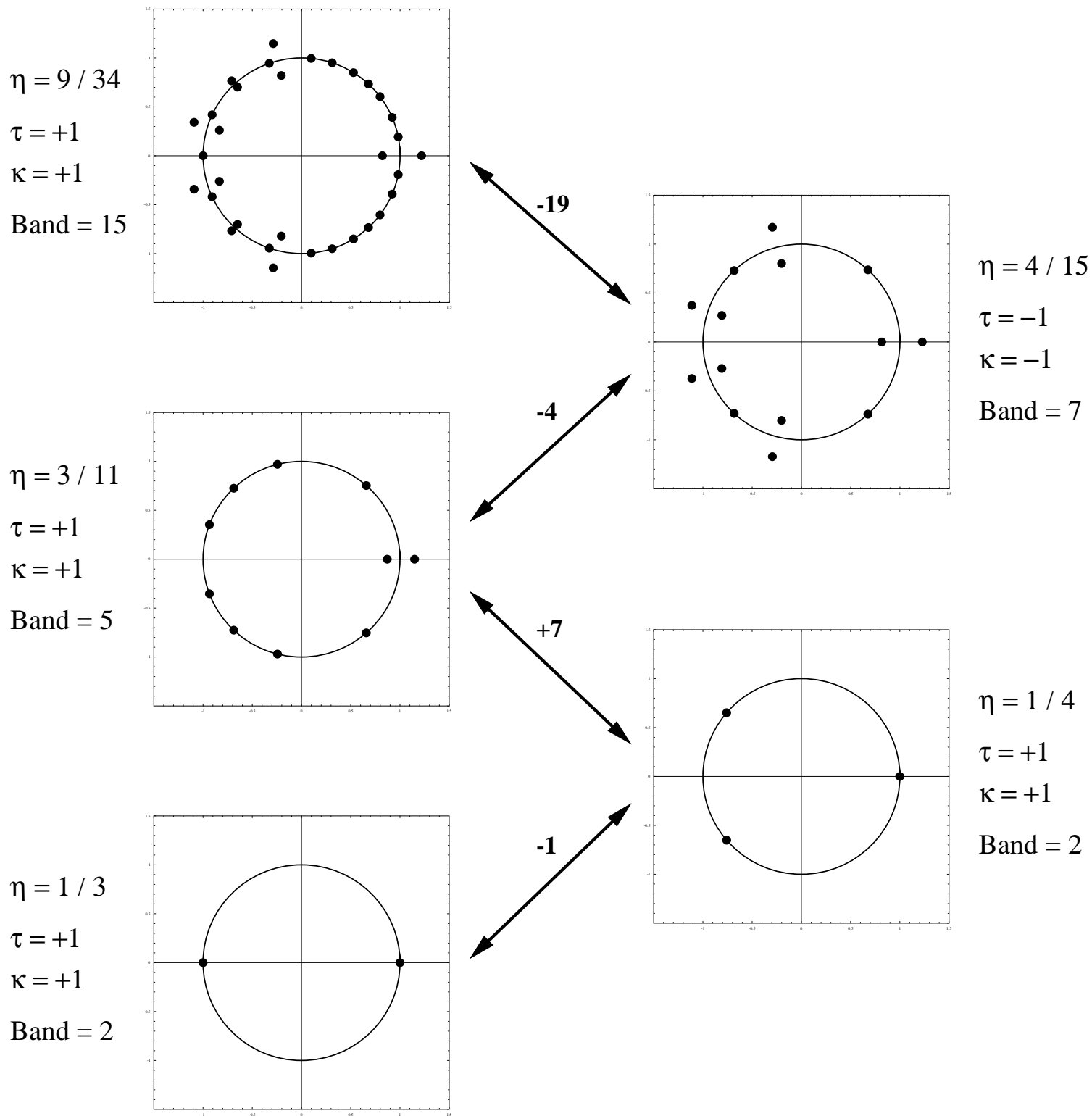

FIG. 2. The root patterns along one branch $\left(J_{7}\right)$ of the hierarchical tree of Fig. 1. Subsequent root patterns along the tree differ by a string whose length is equal to the Hall conductances of the "younger"-one of the two bands. The parities of these strings are in agreement with (23) and are shown as signs in front of string lengths. The band numbers are given as counted from the bottom of the spectrum. For all states shown $\mu=+1$. 


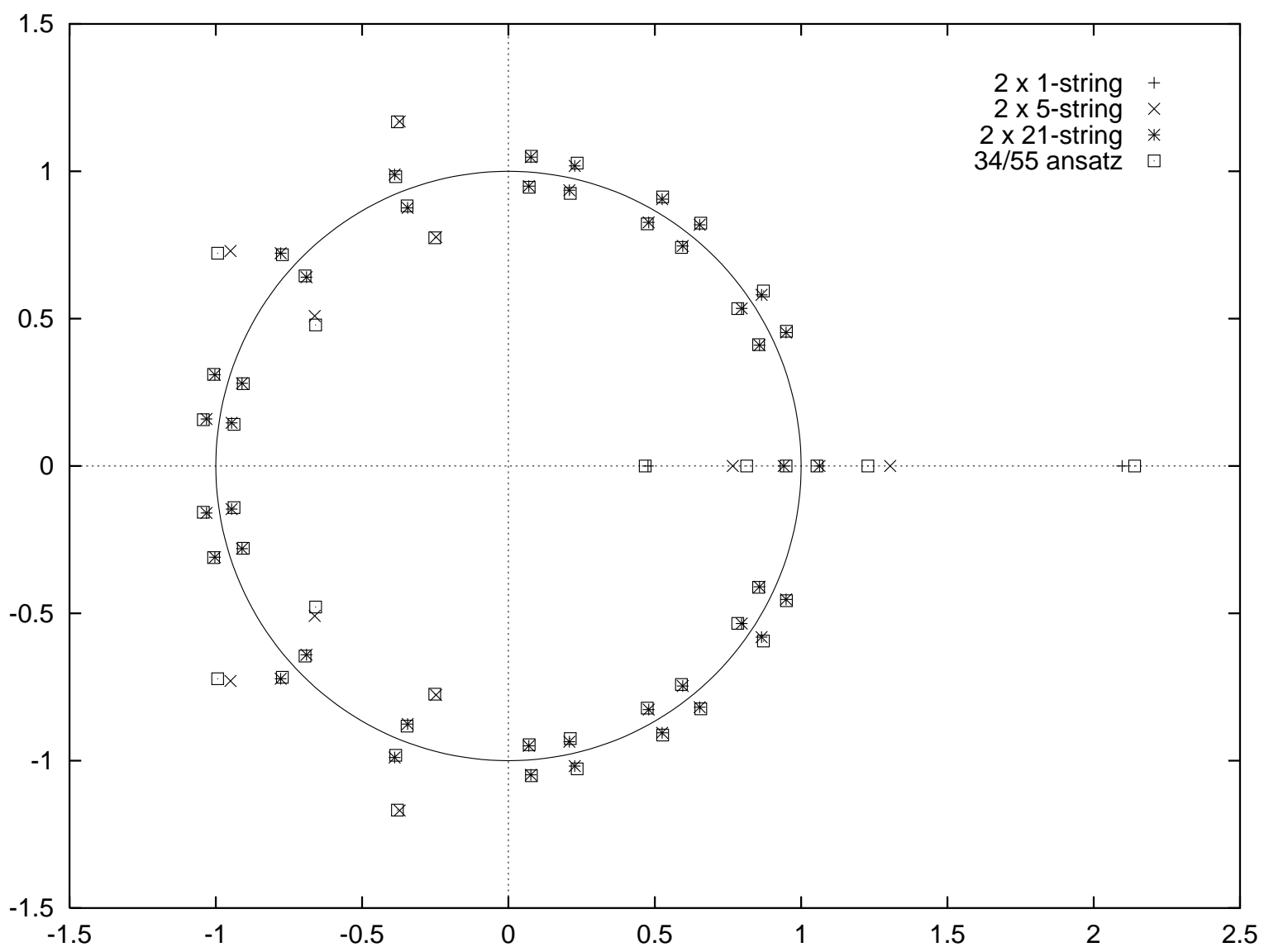

FIG. 3. Shown in the figure are the roots that parameterize the ground state of the Hofstadter problem at flux 34/55. The roots are organized in strings: two of the length $1(+)$, two of the length $5(\times)$ and two of the length $21(*)$. These roots can be approximated by ansatz (34) and are plotted in the Figure as boxes $(\square)$. Strings or more generally roots tend to repel each other. This "splitting" is captured empirically by the expression for $x_{n}$ in (34). 

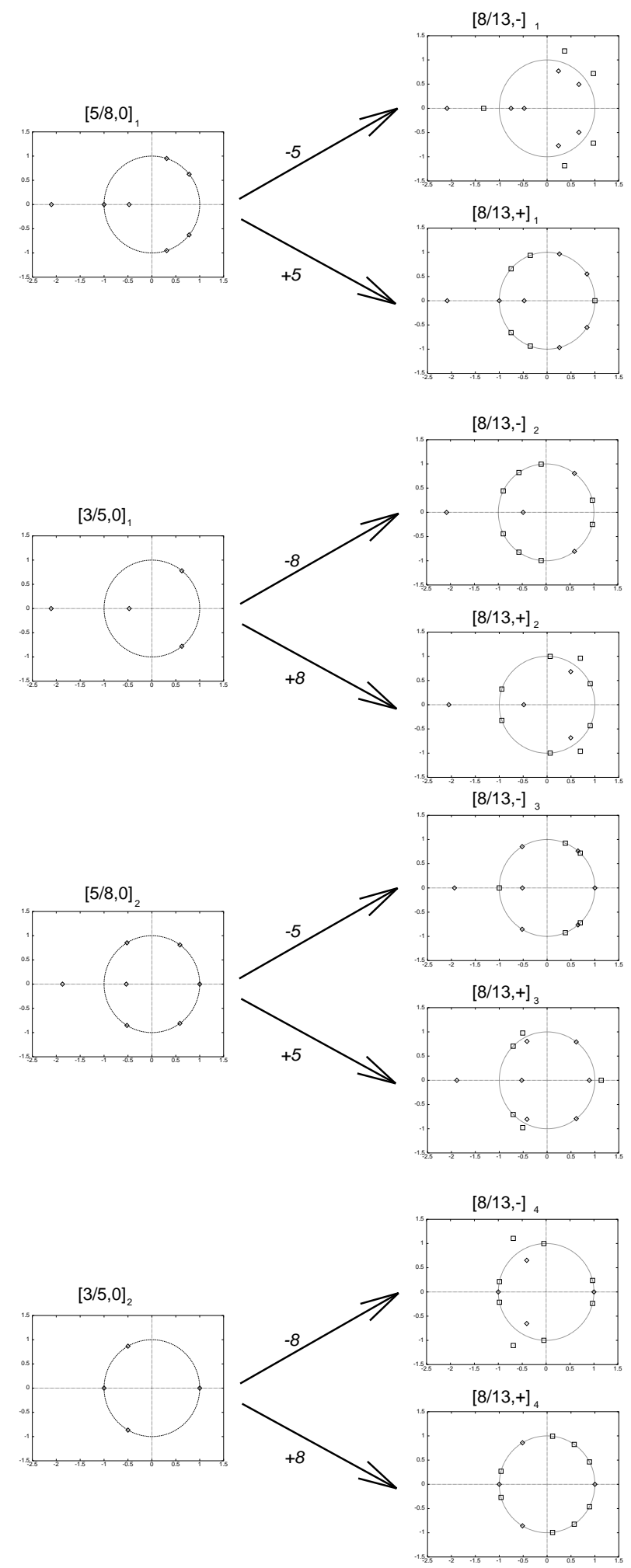
$[8 / 13,-]_{5}$
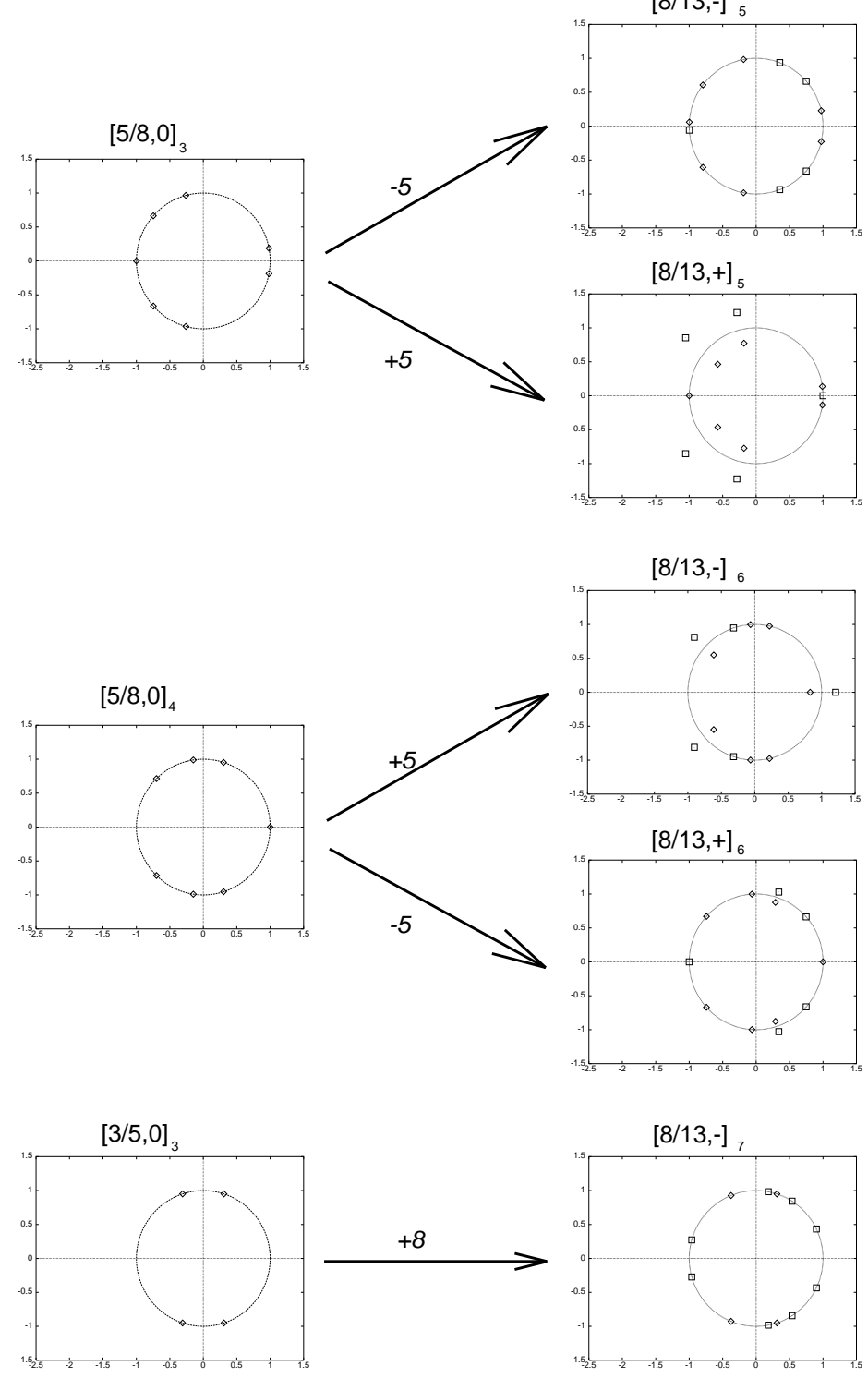

FIG. 4. This figure shows the evolution of root's pattern along the hierarchical tree: root patterns of the generation with the flux $\frac{8}{13}$ are constructed out of previous generations with the flux $\frac{5}{8}$ and $\frac{3}{5}$ ) by adding strings with the lengths 5 or 8 respectively with parities \pm denoted as sign in front of string lengths. The symbol $\left[\frac{P}{Q},-0+\right]_{i}$ denotes a state at the bottom $(-)$, middle $(0)$ or top $(+)$ of the $i$-th band (counted from the bottom of the spectrum) of the generation with a flux $\frac{P}{Q}$. 


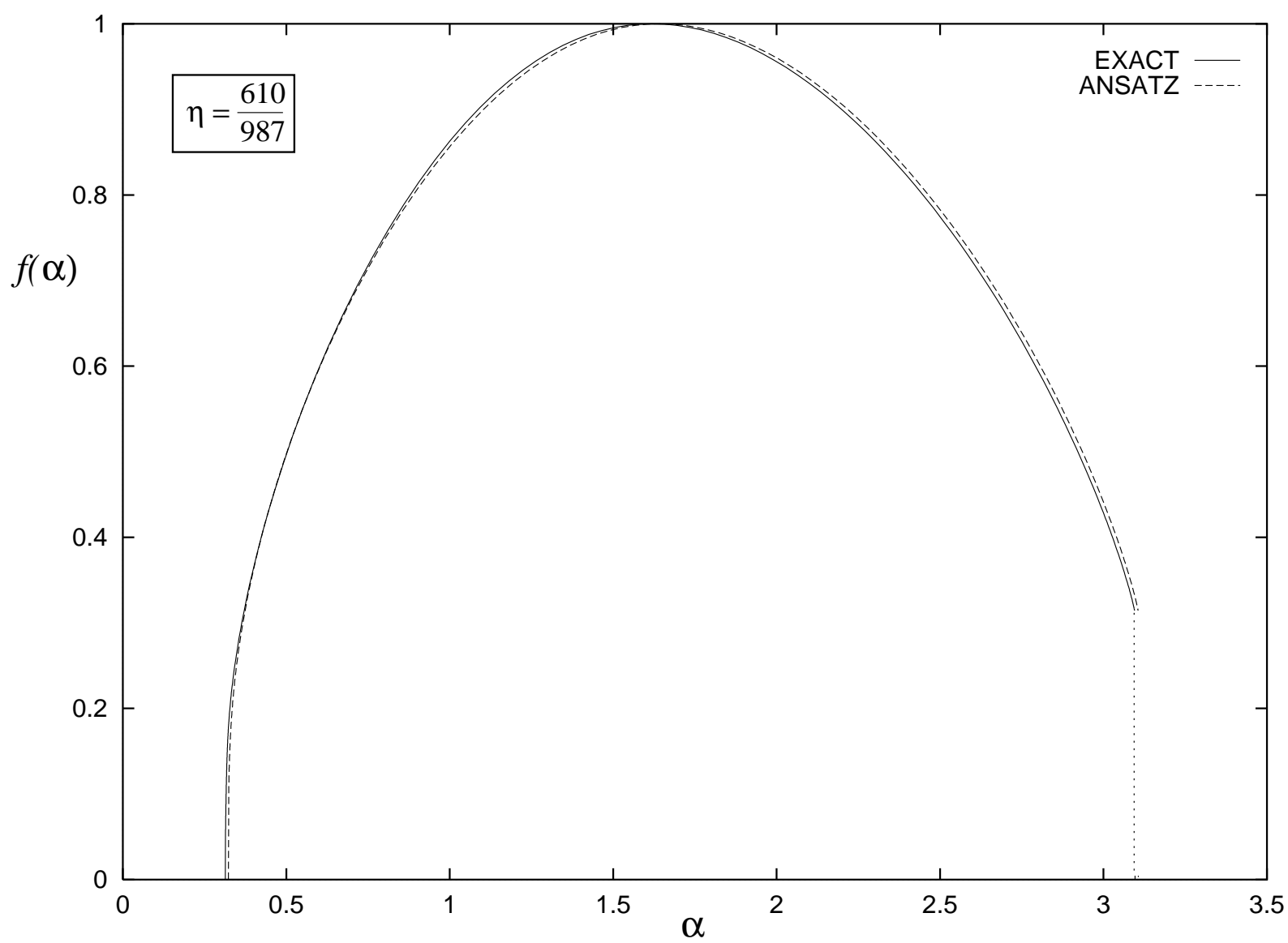

FIG. 5. The function $f(\alpha)$ for the wave function of the bottom of the lowest band. The solid line is an exact result (found numerically), the dashed line is the $f(\alpha)$ of the approximate wave function (34). The support of $f(\alpha)$ is $0.3 \approx \alpha_{\min } \leq \alpha \leq \alpha_{\max } \approx 3.5$. 


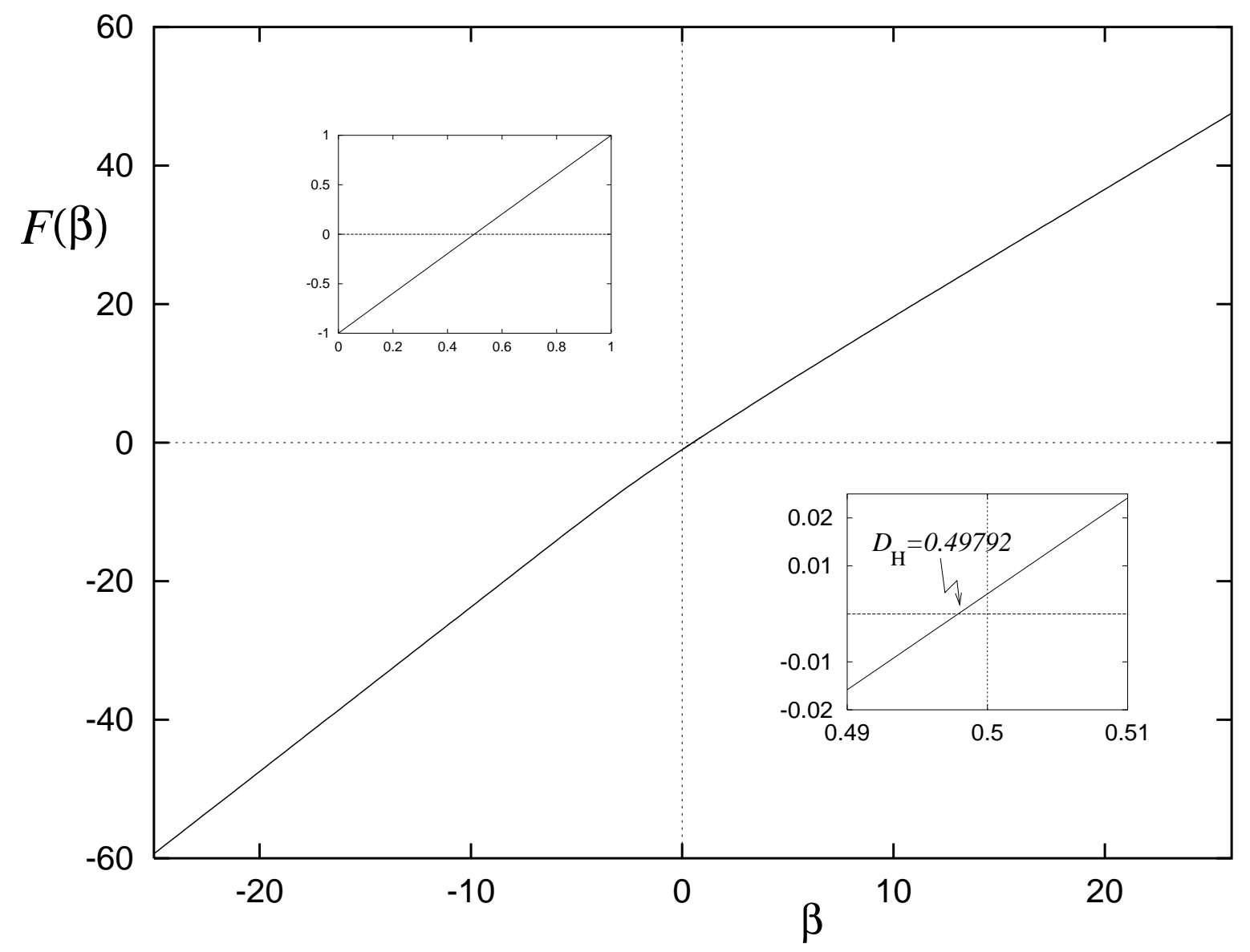

FIG. 6. The free energy $F(\beta)$ is plotted as a function of $\beta$ for the flux equal to the golden mean $\eta=\frac{\sqrt{5}-1}{2}$. The deviation from a straight line indicates multifractality. The leftmost inset zooms into the region $\beta \in[0,1]$. It includes the points $F(\beta=0)=-1$ and $F(\beta=1)=1$ known exactly. The rightmost inset zooms into the region where $F\left(\beta=D_{\mathrm{H}}\right)=0$. $D_{\mathrm{H}}$ is the Hausdorff dimension. The slope of $F$ at this point gives the most probable scaling dimension $\alpha_{0}=\frac{1}{F^{\prime}\left(D_{\mathrm{H}}\right)}$. Although both Hausdorff dimensions and the most probable scaling dimension are numerically close to 0.5 they differ from $1 / 2$ within the numerical error-margin. The error margin $\left(10^{-8}-10^{-7}\right)$ is not visible in the figure. 